3D Diffraction Tomography

DECTRIS Ltd.

+41565002100

www.dectris.com

HREM Research, Inc.

81-493-35-3919

www.hremresearch.com

NanoMEGAS

480-389-6816

www.nanomegasusa.com

Rigaku Corporation

+1-281-362-2300

www.rigaku.com

\section{D Live Cell Imaging}

Abberior Instruments America LLC +15619729300

www.abberior-instruments-america. com

CytoViva, Inc.

1-334-321-3901

www.cytoviva.com

Edge-3d

808.344.5954

www.edge-3d.com

Forth Dimension Displays Ltd.

+44 0 1383-827-950

www.forthdd.com

NTT Advanced Technology

Corporation

www.ntt-at.com

Meadowlark Optics

303-833-4333

www.meadowlark.com

\section{D Measurement}

Olympus

8322437900

https://www.olympus-ims. com/en/microscope/?utm source=microscopy-today\&utm medium=marketing_network\&utm _ campaign=Dec_2020_IE_All\&utm_ content=listing

\section{D Tomography Software \\ DigiSENS \\ www.digisens3d.com \\ XRE \\ +32 (0)93946794 \\ http://xre.be}

\section{6-Axis Positioners}

Aerotech, Inc.

+1-412-963-7470

www.aerotech.com

\author{
Accessories (miscellaneous) \\ APPLIED IMAGE Inc. \\ 585-482-0300 \\ www.appliedimage.com \\ CeramOptec Industries, Inc. \\ 413-525-0600 \\ www.ceramoptec.com
}

Chroma Technology Corp. 800-824-7662

www.chroma.com

Bel-Art Products

www.belart.com

Electro-Optics Technology, Inc. 231-935-4044

www.eotech.com

Ellcie Industries $\mathrm{GmbH}$

+493881755400

www.ellcie.com

Evactron $\AA$ by XEI Scientific, Inc. 650-369-0133

www.evactron.com

Finger Lakes Instrumentation

585-624-3760

www.flicamera.com

ibss Group, Inc.

650-513-1488

www.ibssgroup.com

Microyn Technologies, Inc.

410-967-7118

www.microyntech.com

MTIInstruments/Fullam

www.mtiinstrumen ts.com

Omega Optical, LLC

802-251-7300

www.omegafilters.com

RedWave Technology

203-628-4833

www.redwavetech.com

Spicer Consulting Limited $+44(0) 1234765773$ www.spicerconsulting.com

Valley Design Corp.

978-425-3030

www.valleydesign.com

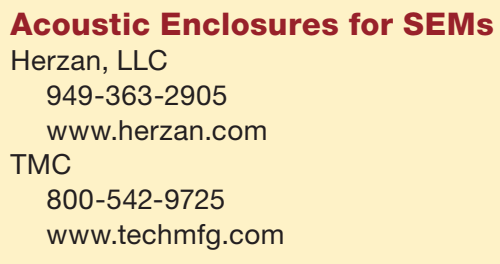

\section{AFM Liquid Imaging,}

SECM, SECCM

Park Systems, Inc.

408-986-1110

www.parksystems.com

\section{AFM Probes}

Bruker Nano Surfaces and Metrology 520-741-1044 ext. 1075 www.bruker.com/nano

Carbon Design Innovations 650-697-7070

www.carbondesigninnovations.com

K-Tek Nanotechnology, LLC

503-598-9828

www.kteknano.com

NanoAndMore USA, Inc.

843-521-1108

www.nanoandmore.com
AFM/SPM Accessories

Advanced Surface Microscopy, Inc. 317-895-5630

www.asmicro.com

AFMWorkshop

888-671-5539

www.afmworkshop.com

A.P.E. Research SRL

$+390402528164$

www.aperesearch.com

Asylum Research, an Oxford Instruments

Company

805-696-6466

www.oxford-instruments.com/AFM

Carbon Design Innovations

650-697-7070

www.carbondesigninnovations.com

K-Tek Nanotechnology, LLC

503-598-9828

www.kteknano.com

Minus K Technology, Inc. 310-348-9656

www.minusk.com

NanoAndMore USA, Inc. 843-521-1108

www.nanoandmore.com

Nanosurf AG

+41619274747

www.nanosurf.com

nPoint, Inc.

608-824-1770

www.npoint.com

Semilab USA LLC

+1-508-647-8411

http://www.semilab.com

Ted Pella, Inc.

800-237-3526

www.tedpella.com

\section{Analytical Balances}

Citizen Scale Inc.

732-744-1440

www.citizeninc.com

\section{Antibodies}

Miltenyi Biotec B.V. \& Co. KG

+49220483060

www.miltenyibiotec.com

\section{Anti-Contamination Systems}

Evactron $\AA$ by XEI Scientific, Inc.

650-369-0133

www.evactron.com

ibss Group, Inc.

650-513-1488

www.ibssgroup.com

\section{Anti-Vibration Devices}

Bel-Art Products

www.belart.com

Circuit Insights LLC

626-201-0488

www.LoopSlooth.com 


\section{A Guide to Microscopy Products}

Herzan, LLC

949-363-2905

www.herzan.com

K-Tek Nanotechnology, LLC 503-598-9828

www.kteknano.com

Minus K Technology, Inc. 310-348-9656 www.minusk.com

Vibration Engineering Consultants +1 831-465-9189

https://vibeng.com/

\section{AP3Windows}

Moxtek, Inc.

801-225-0930

www.moxtek.com

\section{Apertures}

Applied Beams LLC 503-608-7237

www.appliedbeams.com

Agar Scientific Ltd +44 (0)1279215 506

www.agarscientific.com

Clark-MXR, Inc.

734-426-2803

www.cmxr.com

Electron Microscopy Sciences 215-412-8400 www.emsdiasum.com

HTA Photomask

408 452-5500

www.htaphotomask.com

Ladd Research

802-658-4961

www.laddresearch.com

METRIGRAPHICS, LLC 800-261-2557

http://www.metrigraphicsllc.com/

Norsam Technologies, Inc.

505-984-1133

www.norsam.com

Oregon Physics

503-601-0041

www.oregon-physics.com

Reynard Corp.

949-366-8866

www.reynardcorp.com

Ted Pella, Inc.

800-237-3526

www.tedpella.com

\section{Atom Probe}

3D-Micromac AG

+49 371 40043-222

www.3d-micromac.com

CAMECA

+33143346200

www.cameca.com

\section{Atomic Force}

Microscopes (AFMs)

Advanced Research Corporation www.arcnano.com
Advanced Surface Microscopy, Inc. 317-895-5630

www.asmicro.com

AFMWorkshop

888-671-5539

www.afmworkshop.com

AIST-NT

www.aist-nt.com

Anasys Instruments 805-730-3310

www.anasysinstruments.com

Angstrom Science, Inc 805-845-7446

www.angstromscience.com

A.P.E. Research SRL +390402528164

www.aperesearch.com

Asylum Research, an Oxford Instruments Company

805-696-6466

www.oxford-instruments.com/AFM

Bruker AFM Probes

520-741-1044

www.brukerafmprobes.com

Bruker Nano Surfaces and Metrology 520-741-1044 ext. 1075

www.bruker.com/nano

Hitachi High Technologies America, Inc. 800-253-3053

www.hitachi-hightech.com/us

ION-TOF USA, Inc.

845-352-8082

www.iontofusa.com

Mad City Labs, Inc.

608-298-0855

www.madcitylabs.com

Microscopy/Microscopy Education (MME) 972-924-5310

www.MicroscopyEducation.com

MicroSpec On-site

972-924-5310

microspec-onsite.com

NanoMagnetics Instruments Ltd. +441865522989

www.nanomagnetics-inst.com

Nanonics Imaging, Ltd.

866-220-6828

www.nanonics.co.il

Nanoscience Instruments, Inc. 888-777-5573

www.nanoscience.com

Nanosurf AG

+41619274747

www.nanosurf.com

neaspec $\mathrm{GmbH}$

49-176-2320-3996

www.neaspec.com

NenoVision

+420605287732

www.nenovision.com

Park Systems, Inc.

408-986-1110

www.parksystems.com

PI (Physik Instrumente), LP

508-832-3456

www.pi-usa.us/microscopy
Quantum Design, Inc.

858-481-4400

https://www.qdusa.com/

Semilab USA LLC

+1-508-647-8411

http://www.semilab.com

SPECS

+49-30-4678240

www.specs.com

Used-Line

845-287-0066

Used-Line.com

WITec GmbH

+49(0)731 140700

www.witec.de

\section{Auger Microscopes}

Physical Electronics

952-828-6100

www.phi.com

\section{Autofocus Systems}

GIGAmacro

415-841-3322

www.gigamacro.com

Motion X Corporation

805-968-2001

www.motionxcorp.com

nPoint, Inc.

608-824-1770

www.npoint.com

PIEZOCONCEPT

+33(0)478742456

www.piezoconcept.com

Pixelink

1-833-247-1211

www.pixelink.com

TOFRA, Inc.

650-387-5497

www.tofrainc.net/

\section{Automation/Robotic}

Equipment

Aerotech, Inc

+1-412-963-7470

www.aerotech.com

BrandTech Scientific, Inc.

(888) 522-2726

www.brandtech.com

Microscopy Innovations

715-384-3292

www.microscopyinnovations.com

Motion X Corporation

805-968-2001

www.motionxcorp.com

TCG, Inc.

925-240-5210

www.tcg-rep.com

Velmex, Inc.

585-657-6151

www.velmex.com

Voxa

415-858-0393

http://projectvoxa.com 


\section{A Guide to Microscopy Products}

\section{Backscattered Electron \\ Detectors}

Photonic Science and Engineering +44 (0) 1424444883 www.photonicscience.com

PNDetector $\mathrm{GmbH}$ +4989 309087-100 www.pndetector.de

PNSensor GmbH

(49) 8930908713

www.pnsensor.de

Seron Technologies, Inc. 82-031-349-1411 www.serontech.co.kr

X-Spectrum GmbH +4915731711472 https://x-spectrum.de/

\section{Beam Splitters}

Access Optics 918-294-1234 www.accessoptics.com

Chroma Technology Corp. 800-824-7662 www.chroma.com

DayOptics, Inc. +8659183215681 www.dayoptics.com

Deposition Sciences, Inc. 707-573-6700 www.depsci.com

Ealing Catalog 800-295-3220 www.ealingcatalog.com

Evaporated Coatings, Inc. 215-659-3080 www.evaporatedcoatings.com

Guernsey Coating Laboratories, Inc. 805-642-1508 www.guernseycoating.com

Iridian Spectral Technologies Ltd. 613-741-4513 www.iridian.ca

Laser 2000 (UK) Ltd. +44 (0) 01933461666 www.laser2000.co.uk

Laser Components USA, Inc. (603) 8217040 www.lasercomponents.com

MLD Technologies, LLC (650) 938-4485 www.mldtech.com

Omega Optical, LLC 802-251-7300 www.omegafilters.com

Perkins Precision Developments, LLC 970-785-2706 www.perkinsprecision.com

Photometrics 520-889-9933 www.photometrics.com

Precision Glass \& Optics (PG\&O®) 714-540-0126 www.pgo.com

Rainbow Research Optics, Inc. (RROI) 303-371-3000
RR-Optics.com Reynard Corp. 949-366-8866

www.reynardcorp.com

Special Optics

973-366-7289

www.specialoptics.com

UQG (Lab Glasses)

+44 (0) 1223420329

www.uqgoptics.com

\section{Benchtop Micro CT}

Angstrom Scientific, Inc. 201-962-7222

www.angstrom.us

\section{Benchtop SEM \\ COXEM CO., LTD \\ +82-42-861-1686 \\ www.coxem.com \\ Delong America \\ 1-866-335-6648 \\ www.lv-em.com}

Electron Optics Instruments LLC 973-220-8689

www.eoillc.com

Hitachi High Technologies America, Inc. 800-253-3053

www.hitachi-hightech.com/us

H.K.N. Inc.

408-722-8478

www.xvor.co.jp

McCrone Microscopes \& Accessories 630-887-7100

www.mccrone.com

Nanolmages, LLC

866-601-6266

www.nanoimages.com

Seron Technologies, Inc.

82-031-349-1411

www.serontech.co.kr

\section{Biology and Cytology}

ARGOLIGHT

+33 (0)564 310850

www.argolight.com

Motic Instruments, Inc. 604-303-9033

https://moticmicroscopes.com/

Psylotech Inc.

+1-847-328-7100

www.psylotech.com

Teledyne Photometrics 520-889-9933

photometrics.com

Bioreactor Cell Culture System

Glen Mills Inc.

973-777-0777

www.GLENMILLS.com

Books and Textbooks

ASM International

440-338-5151

asminternational.org
Royal Microscopical Society

01865265760

www.rms.org.uk

\section{Calibration and}

Reference Standards

Advanced Surface Microscopy, Inc. 317-895-5630

www.asmicro.com

Agar Scientific Ltd +44 (0)1279 215506 www.agarscientific.com

APPLIED IMAGE Inc.

585-482-0300

www.appliedimage.com

ARGOLIGHT

+33 (0)564 310850

www.argolight.com

ATAGO U.S.A., Inc.

425-637-2107

http://www.atago-usa.com

Avian Rochester, LLC

+1 585 259-5956

avianrochester.com

Axiom Optics

617-221-6636

www.axiomoptics.com

Datacolor Inc.

800-982-6499

www.datacolor.com

Gamma Scientific

858-279-8034

www.gamma-sci.com

Geller MicroAnalytical Laboratory, Inc. 978-887-7000

www.gellermicro.com

HEIDENHAIN Corporation

847-490-1191

www.heidenhain.us

Hirox-USA, Inc.

201-342-2600

www.hirox-usa.com

McCrone Atlas of Microscopic Particles 630-887-7100

www.mccroneatlas.com

Microscopy/Microscopy Education (MME) 972-924-5310

www.MicroscopyEducation.com

Modem Microscopy

630-887-7100

www.modernmicroscopy.com

Norsam Technologies, Inc.

505-984-1133

www.norsam.com

Small World, LLC

703-849-1492

www.small-world.net

Ted Pella, Inc.

800-237-3526

www.tedpella.com

\section{Camera/Digital Camera Systems}

ACCU-SCOPE, Inc.

631-864-1000

www.accu-scope.com 


\section{A Guide to Microscopy Products}

Alrad Imaging

+44 (0)1635 30345

www.alrad.com

Basler AG

+49-4102-463-500

www.baslerweb.com

BOCK OPTRONICS INC.

416-674-2804

www.bockoptronics.ca

Dage-MTI

219-872-5514

www.dagemti.com

Datacolor Inc.

800-982-6499

www.datacolor.com

Diffraction Limited

+1-613-225-2732

https://www.diffractionlimited.com

Direct Electron, LP

+1 858-384-0291

www.directelectron.com

E. A. Fischione Instruments, Inc.

724-325-5444

www.fischione.com

EMSIS GmbH

+492512979620

www.emsis.eu

Excel Technologies

860-741-3435

www.exceltechnologies.com

Gatan

925-463-0200

www.gatan.com

Hamamatsu Corporation

908-231-0960

www.hamamatsu.com

JAI

1-800-445-5444

www.jai.com

JENOPTIK Optical Systems

561-881-7400

www.jenoptik.com

Lambert Instruments BV

+31 (0) 505018461

www.lambertinstruments.com

Lumenera Corporation

613-736-40 77

www.lumenera.com

Mager Scientific, Inc.

734-426-3885

www.magersci.com

Martin Microscope Company

864-242-3424

www.MartinMicroscope.com

MICROS Produktions-und HandelsgmbH www.micros.at

Microyn Technologies, Inc.

410-967-7118

www.microyntech.com

Motic Instruments, Inc.

604-303-9033

https://moticmicroscopes.com/

Nili Walp

860-355-3776

www.visioneng.us
Photometrics

520-889-9933

www.photometrics.com

Photon etc.

514-385-9555

www.photonetc.com

Photonic Science and Engineering

+44 (0) 1424444883

www.photonicscience.com

Photron

858-684-3555

www.photron.com

Pixelink

1-833-247-1211

www.pixelink.com

PNDetector $\mathrm{GmbH}$

+4989 309087-100

www.pndetector.de

Princeton Infrared Technologies, Inc. 609-917-3380

www.princetonirtech.com

Qlmaging

604-530-5800

www.Qimaging.com

Raptor Photonics

+442828270141

www.raptorphotonics.com

ResAlta Research Technologies Corp. 303-748-4346

www.resaltatech.com

Scientific Instruments \& Applications, Inc.

770-232-7785

www.sia-cam.com

Seiwa Optical America Inc.

408-844-8008

www.seiwaamerica.com

Semion

503-332-9668

www.semionco.com

SENSORS UNLIMITED INC., A PART OF

COLLINS AEROSPACE

609-333-8200

www.sensorsinc.com

Stanford Photonics, Inc.

650-969-5991

www.stanfordphotonics.com

CANON MEDICAL, Video Sensing

Division

810-357-5022

https://us.medical.canon/VSD

Teledyne Photometrics

520-889-9933

photometrics.com

TRU-Vu Monitors, Inc.

847-259-2344

www.TRU-VuMonitors.com

TVIPSGmbH

49-89-8506567

www.tvips.com

UVP LLC

909-946-3197

www.uvp.com

Vision Engineering, Inc.

860-355-3776

www.visioneng.us
Vee Gee Scientific 800-423-8842

www.veegee.com

VEROPTICS

1-800-939-6627

www.veroptics.com

ZEISS

1-800-233-2343

www.zeiss.com/microscopy

\section{Cameras, CCD}

Alrad Imaging

+44 (0)1635 30345

www.alrad.com

Andor Technology

+442890237126

www.andor.oxinst.com

Basler AG

+49-4102-463-500

www.baslerweb.com

Deben

+44 (0) 1359244 870; 12014105028

www.deben.co.uk

Diffraction Limited

+1-613-225-2732

https://www.diffractionlimited.com

EMSIS GmbH

+49251297962 0

www.emsis.eu

Finger Lakes Instrumentation

585-624-3760

www.flicamera.com

Hamamatsu Corporation

908-231-0960

www.hamamatsu.com

HORIBA Scientific

732-494-8660

www.horiba.com/scientific

Lumenera Corporation

613-736-4077

www.lumenera.com

Mightex Systems

1-925-218-1885

www.mightexsystems.com

Pembroke Instruments, LLC

415-860-4127

https://pembrokeinstruments.com

Photometrics

520-889-9933

www.photometrics.com

PNDetector $\mathrm{GmbH}$

+4989 309087-100

www.pndetector.de

Photonic Science and Engineering

+44 (0) 1424444883

www.photonicscience.com

Qlmaging

604-530-5800

www.Qimaging.com

Raptor Photonics

+442828270141

www.raptorphotonics.com 
Scientific Instruments \& Applications, Inc. 770-232-7785

www.sia-cam.com

Seiwa Optical America Inc. 408-844-8008 www.seiwaamerica.com

SPOT Imaging Solutions 586-731-6000 www.spotimaging.com

Stanford Photonics, Inc. 650-969-5991 www.stanfordphotonics.com

Teledyne Princeton Instruments 609-587-9797 princetoninstruments.com

Z\&Z Optoelectronics Tech Co., Ltd. 86-28-85139768

www.zzoptic.com

\section{Cameras, CMOS}

Alrad Imaging +44 (0)1635 30345

www.alrad.com

Basler AG $+49-4102-463-500$ www.baslerweb.com

CANON MEDICAL, Video Sensing Division

810-357-5022

https://us.medical.canon/VSD

Dage-MTI

219-872-5514

www.dagemti.com

Diffraction Limited

+1-613-225-2732

https://www.diffractionlimited.com

Dunwell Tech

888-668-2442

http://dinolite.us/

EMSIS GmbH

+492512979620

www.emsis.eu

Finger Lakes Instrumentation

585-624-3760

www.flicamera.com

GIGAmacro

415-841-3322

www.gigamacro.com

Hamamatsu Corporation 908-231-0960

www.hamamatsu.com

JAI

1-800-445-5444

www.jai.com

Lumenera Corporation

613-736-4077

www.lumenera.com

Microscope.com

540-904-0880

www.microscope.com

Mightex Systems

1-925-218-1885

www.mightexsystems.com

Navitar

585-359-4000

www.navitar.com
Pembroke Instruments, LLC 415-860-4127

https://pembrokeinstruments.com

PCO-TECH Inc.

+1 (248) 2768820

www.pco-tech.com

Photonic Science and Engineering +44 (0) 1424444883

www.photonicscience.com

Photometrics

520-889-9933

www.photometrics.com

Photron

858-684-3555

www.photron.com

Pixelink

1-833-247-1211

www.pixelink.com

QImaging

604-530-5800

www.Qimaging.com

SPOT Imaging Solutions

586-731-6000

www.spotimaging.com

Stanford Photonics, Inc.

650-969-5991

www.stanfordphotonies.com

Teledyne Photometrics

520-889-9933

photometrics.com

Teledyne Princeton Instruments 609-587-9797

princetoninstruments.com

TRU-Vu Monitors, Inc.

847-259-2344

www.TRU-VuMonitors.com

\section{Cameras, EMCCD}

Andor Technology +442890237126

www.andor.oxinst.com

Hamamatsu Corporation 908-231-0960 www.hamamatsu.com

Laser 2000 (UK) Ltd. +44 (0) 01933461666 www.laser2000.co.uk

Photometrics 520-889-9933

www.photometrics.com

QImaging

604-530-5800

www.Qimaging.com

Raptor Photonics

+442828270141

www.raptorphotonics.com

Stanford Photonics, Inc.

650-969-5991

www.stanfordphotonics.com

Teledyne Princeton Instruments 609-587-9797

princetoninstruments.com

\section{Cameras, Intensified CCD}

Andor Technology

+442890237126

www.andor.oxinst.com

Archer OpTx, Inc.

972-722-1064

www.archeroptx.com

Lambert Instruments BV

+31 (0) 505018461

www.lambertinstruments.com

PCO-TECH Inc.

+1 (248) 2768820

www.pco-tech.com

Photonic Science and Engineering +44 (0) 1424444883

www.photonicscience.com

Photometrics

520-889-9933

www.photometrics.com

QImaging

604-530-5800

www.Qimaging.com

Scientific Instruments \& Applications, Inc. 770-232-7785

www.sia-cam.com

Teledyne Princeton Instruments 609-587-9797

princetoninstruments.com

\section{Cameras, Hybrid Pixel}

Technology

DECTRIS Ltd.

+41565002100

www.dectris.com

\section{Cantilevers}

K-Tek Nanotechnology, LLC 503-598-9828

www.kteknano.com

Seiwa Optical America Inc. 408-844-8008

www.seiwaamerica.com

\section{Carbon Coaters}

Anatech USA

775-657-8685

www.anatechusa.com

Denton Vacuum

856-439-9111

www.dentonvacuum.com

element $\mathrm{Pi}$

833-314-1593

www.elementpi.com

Ladd Research

802-658-4961

www.laddresearch.com

Quorum Technologies, Ltd. $+44(0) 1323810981$

www.quorumtech.com

Ted Pella, Inc.

800-237-3526

www.tedpella.com 


\section{A Guide to Microscopy Products}

\author{
Carbon Nanotube Probes for AFM \\ Carbon Design Innovations \\ 650-697-7070 \\ www.carbondesigninnovations.com \\ K-Tek Nanotechnology, LLC \\ 503-598-9828 \\ www.kteknano.com
}

\section{Centrifuges \\ PRO Scientific}

www.proscientific.com

Used-Line

845-287-0066

Used-Line.com

\section{Chemicals (See Fixatives and Chemicals)}

\section{Cleanliness Inspection \\ Olympus}

8322437900

https://www.olympus-ims.

com/en/microscope/?utm source=microscopy-today\&utm _ medium=marketing_network\&utm _ campaign=Dec_2020_IE_All\&utm_ content=listing

\section{Cleaving}

LatticeGear LLC +1 (503) 828-0400

www.latticegear.com

\section{Coating Supplies}

Microscope.com 540-904-0880

www.microscope.com

Technotrade International, Inc. 603-622-5011 www.technotradeinc.com

Ted Pella, Inc.

800-237-3526

www.tedpella.com

\section{Coating Systems}

Kurt J. Lesker Company 412-387-9200

www.lesker.com

Nanolmages, LLC 866-601-6266 www.nanoimages.com

Nor-Cal Products by Pfeiffer Vacuum 800-824-4166 www.n-c.com

Pantec Engineering AG +423377 1333 www.pantec-medicallaser.com

Quorum Technologies, Ltd. $+44(0) 1323810981$ www.quorumtech.com
SPI Supplies

610-436-5400

www.2spi.com

Ted Pella, Inc.

800-237-3526

www.tedpella.com

\section{Coating Thickness Gauge}

Filmetrics, A KLA Company

858-573-9300

www.filmetrics.com

Kett US

www.kett.com

Phillip Technologies

864-277-1560

www.PhillipTech.com

\section{Cold Sputtering Equipment}

Denton Vacuum

856-439-9111

www.dentonvacuum.com

element $\mathrm{Pi}$

833-314-1593

www.elementpi.com

Quorum Technologies, Ltd.

$+44(0) 1323810981$

www.quorumtech.com

Ted Pella, Inc.

800-237-3526

www.tedpella.com

\section{Colloidal Gold}

Electron Microscopy Sciences 215-412-8400 www.emsdiasum.com

Nanoprobes, Inc. 1-877-447-6266, (631) 205-9490

www.nanoprobes.com

Ted Pella, Inc.

800-237-3526

www.tedpella.com

\section{Compact SEM}

Seron Technologies, Inc. 82-031-349-1411 www.serontech.co.kr

\section{Conductive Adhesives for SEM}

Ted Pella, Inc.

5303395049

www.tedpella.com

\section{Confocal Microscopes}

attocube systems +49-89-2877-809-0 www.attocube.com

Boston Electronics 617-566-3821

www.boselec.com

Bruker Optics

978-663-3660

www.bruker.com
Bruker Fluorescence Microscopy 608-662-0022

http://www.bruker.com

Bruker Nano Surfaces and Metrology 520-741-1044 ext. 1075 www.bruker.com/nano

CrestOptics www.crestopt.com

Excel Technologies 860-741-3435 www.exceltechnologies.com

Forth Dimension Displays Ltd. +44 0 1383-827-950 www.forthdd.com

Harrick Scientific Products, Inc. www.harricksci.com

Holo/Or Ltd. +972-8-9409687 www.holoor.co.il/

Leica Microsystems 800-248-0123 www.leica-microsystems.com

NTT Advanced Technology Corporation www.ntt-at.com

Microscopy/Microscopy Education (MME)

972-924-5310

www.MicroscopyEducation.com

PicoQuant GmbH +49-(0)30-6392-6929 www.picoquant.com

Phaseview +33 954030543 www.phaseview.com

VEROPTICS 1-800-939-6627 www.veroptics.com

Vutara

801-512-2664

www.vutara.com

WITec GmbH $+49(0) 731140700$ www.witec.de

ZEISS

1-800-233-2343

www.zeiss.com/microscopy

\section{Consulting}

Electron Optica, Inc. 415-706-1557 www.electronoptica.com

Field Management Services, Inc. 212-628-6860 x. 205 www.fms-corp.com

GIGAmacro 415-841-3322 www.gigamacro.com

Imaging and Analysis, LLC 651-788-2261 www.imagingandanalysis.com

Nanomechanics, Inc., a KLA Tencor Company 865-978-6490 www.nanomechanicsinc.com 


\section{A Guide to Microscopy Products}

\section{NANOVIZZ}

www.nanovizz.com

Optikos Corporation

617-354-7557

www.optikos.com

Pantec Engineering AG

+423377 1333

www.pantec-medicallaser.com

Particle Technology Labs 630-969-2703

www.particletechlabs.com

Polylnsight LLC www.polyinsight.com

RedWave Technology 203-628-4833 www.redwavetech.com

Visage Imaging 858-356-5517 www.visageimaging.com

Wolfram Research, Inc. 217-398-0700 https://www.wolfram.com

\section{Contract Research}

Applied Beams LLC 503-608-7237 www.appliedbeams.com

Chemlmage Corporation www.chemimage.com

Electron Optica, Inc. 415-706-1557 www.electronoptica.com

NANOVIZZ www.nanovizz.com

Optikos Corporation 617-354-7557 www.optikos.com

Pantec Engineering AG +423377 1333 www.pantec-medicallaser.com

Particle Technology Labs 630-969-2703 www.particletechlabs.com

Polylnsight LLC www.polyinsight.com

\section{Cooling Systems}

Linkam Scientific Instruments +44 (0) 1737363476 www.linkam.co.uk

Psylotech Inc.

+1-847-328-7100

www.psylotech.com

\section{Corrective Microscopy Systems \\ FEI Munich GmbH +49898956620 www.fei.com}

\section{Correlative Microscopy DELMIC BV}

+31(0)157440158

www.delmic.com
Dune Sciences, Inc. 541-359-1959 www.dunesciences.com

Hitachi High Technologies America, Inc. 800-253-3053

www.hitachi-hightech.com/us

Hummingbird Scientific 360-252-2737 www.hummingbirdscientific.com

Linkam Scientific Instruments +44 (0) 1737363476 www.linkam.co.uk

Nanoprobes, Inc. 1-877-447-6266, (631) 205-9490 www.nanoprobes.com

NenoVision +420605287732 www.nenovision.com

Phase Focus Ltd +441142866377 www.phasefocus.com

Quantum Design, Inc. 858-481-4400 https://www.qdusa.com/

Renishaw plc. 847-286-9953 www.renishaw.com/raman

RMC Boeckeler 1-520-745-0001 www.rmcboeckeler.com

Tescan 724-772-7433 www.tescan.com ZEISS

1-800-233-2343

www.zeiss.com/microscopy

\section{Courses/Workshops}

AFMWorkshop 888-671-5539 www.afmworkshop.com

ASM International 440-338-5151 asminternational.org

Boeckeler Instruments, Inc. 520-745-0001 www.boeckeler.com

Electron Microscopy Sciences 215-412-8400 www.emsdiasum.com

Hooke College of Applied Sciences 630-887-7100 www.hookecollege.com

Lehigh Microscopy School 610-758-1112 www.lehigh.edu/microscopy

McCrone Research Institute 312-842-7100 www.mcri.org

McCrone Research Institute, Chicago 312-842-7100 www.mcri.org

Microscopy/Microscopy Education (MME) 972-924-5310 www.MicroscopyEducation.com
MicroSpec On-site

972-924-5310

microspec-onsite.com

\section{Critical Point Dryers}

Denton Vacuum

856-439-9111

www.dentonvacuum.com

Electron Microscopy Sciences 215-412-8400

www.emsdiasum.com

Quorum Technologies, Ltd. $+44(0) 1323810981$ www.quorumtech.com

Tousimis

301-881-2450

www.tousimis.com

\section{Cryo EM Sample Equipment}

Angstrom Scientific, Inc.

201-962-7222

www.angstrom.us

\section{Cryoequipment}

Boeckeler Instruments, Inc. 520-745-0001 www.boeckeler.com

ColdEdge Technologies 610-628-6363 www.coldedgetech.com

Cryofab, Inc. 908-686-3636 www.cryofab.com

DELMIC BV +31(0)157440158 www.delmic.com

Kammrath \& Weiss (516) 313-9742 https://www.kammrath-weiss.com/en/

\section{MiTeGen}

1-607-266-8877

www.mitegen.com

Protochips 919-377-0812 www.protochips.com

Quorum Technologies, Ltd. $+44(0) 1323810981$ www.quorumtech.com

ULVAC Technologies, Inc. 978-686-7550 www.ulvac.com

\section{Cryostats}

attocube systems +49-89-2877-809-0 www.attocube.com

Cryofab, Inc. 908-686-3636 www.cryofab.com

CRYO Industries of America, Inc. 603-621-9957 www.cryoindustries.com 
Janis Research Company, LLC 781-491-0888

www.janis.com

Lake Shore Cryotronics

781-491-0888

www.lakeshore.com/janis

Linkam Scientific Instruments +44 (0) 1737363476

www.linkam.co.uk

\section{Cryotransfer Equipment (EM)}

Cryofab, Inc.

908-686-3636

www.cryofab.com

E. A. Fischione Instruments, Inc. 724-325-5444

www.fischione.com

Gatan

925-463-0200

www.gatan.com

Quorum Technologies, Ltd. $+44(0) 1323810981$

www.quorumtech.com

\section{Cryo-Ultramicrotome}

Boeckeler Instruments, Inc. 520-745-0001

www.boeckeler.com

Micro Star Technologies

800-533-2509

www.microstartech.com

RMC Boeckeler

1-520-745-0001

www.rmcboeckeler.com

\section{Crystallographic Mapping}

neaspec $\mathrm{GmbH}$

49-176-2320-3996

www.neaspec.com

Oxford Instruments America, Inc.

800-447-4717

www.oxford-instruments.com

\section{Custom Courses in Microscopy}

McCrone Research Institute

312-842-7100

www.mcri.org

\section{Cut-Off Machines}

3D-Micromac AG

+49371 40043-222

www.3d-micromac.com

Struers Inc.

440-871-0071

www.struers.com

\section{Databases}

ASM International 440-338-5151

asminternational.orgenvir Cell Image Library

(858) 534-0276

http://www.cellimagelibrary.org/home
Exprodo Software

562-275-1786

www.exprodo.com/calpendo

International Centre for Diffraction Data (ICDD)

610-325-9814

www.icdd.com

\section{Desiccators}

Geller MicroAnalytical Laboratory, Inc. 978-887-7000 www.gellermicro.com

Ted Pella, Inc. 800-237-3526

www.tedpella.com

\section{Detectors}

Applied Spectra, Inc. 510-657-7679

www.appliedspectra.com

Applied Beams LLC 503-608-7237

www.appliedbeams.com

Bruker Corporation

608-276-3000

www.bruker.com

Circuit Insights LLC

626-201-0488

www.LoopSlooth.com

Deben

+44 (0) 1359244 870; 12014105028

www.deben.co.uk

Electro-Optics Technology, Inc.

231-935-4044

www.eotech.com

Hamamatsu Corporation

908-231-0960

www.hamamatsu.com

IXRF, Inc.

512-386-6100

www.ixrfsystems.com

J. Kraft Microscopy Services, Inc.

716-592-4402

jkraftmicro.com

Laser Components USA, Inc. (603) 8217040

www.lasercomponents.com

Marktech Optoelectronics, Inc. (518) 956-2980

https://www.marktechopto.com

MAX Detector Repair Group LLC 608-829-0110

maxdetector.com

M.E. Taylor Engineering, Inc. 301-975-9798 www.semsupplies.com

Nextteq LLC

877-312-2333

www.nextteq.com

Opto Diode Corporation, An ITW

Company

805-499-0335

www.optodiode.com
OSI Optoelectronics Inc. +1 310-355-2812

www.osioptoelectronics.com

PNSensor $\mathrm{GmbH}$

(49) 8930908713

www.pnsensor.de

RaySpec Ltd

+44 1628 5330-60

www.rayspec.co.uk

SII Nanotechnology USA, Inc. www.siintusa.com

Spectronics Corporation 516-333-4840 www.spectroline.com

Stanford Photonics, Inc. 650-969-5991 www.stanfordphotonics.com

Teledyne Judson Technologies 215-368-6900 www.teledynejudson.com

X-Spectrum GmbH +4915731711472 https://x-spectrum.de/

Z\&Z Optoelectronics Tech Co., Ltd. 86-28-85139768

www.zzoptic.com

\section{Detectors, hybrid pixel} DECTRIS Ltd.

$+41565002100$

www.dectris.com

\section{Diamond Knives}

Boeckeler Instruments, Inc. 520-745-0001 www.boeckeler.com

Delaware Diamond Knives, Inc. 800-222-5143

www.ddk.com

DiATOME U.S. 2154128390 www.emsdiasum.com

Micro Star Technologies 800-533-2509 www.microstartech.com

Norsam Technologies, Inc. 505-984-1133 www.norsam.com

RMC Boeckeler 1-520-745-0001 www.rmcboeckeler.com

Ted Pella, Inc. 800-237-3526 www.tedpella.com

\section{Diamond Scribing Tools}

LatticeGear LLC

+1 (503) 828-0400

www.latticegear.com

Minitool, Inc.

408-395-1585

www.minitoolinc.com

Ted Pella, Inc. 800-237-3526

www.tedpella.com 
Diamond Wire Saws

Delaware Diamond Knives, Inc. 800-222-5143

www.ddk.com

TCG, Inc.

925-240-5210

www.tcg-rep.com

\section{Differential Interference \\ Contrast}

Microscopy/Microscopy Education (MME) 972-924-5310

www.MicroscopyEducation.com

\section{Digital Archiving/Data Storage}

Cadmet Inc

800-543-7282

www.cadmet.com

Norsam Technologies, Inc. 505-984-1133

www.norsam.com

Quartz Imaging Corporation 604-488-3911 www.quartzimaging.com

ResAlta Research Technologies Corp. 303-748-4346

www.resaltatech.com

Digital Holographic Microscopes

NanoAndMore USA, Inc.

843-521-1108

www.nanoandmore.com

Digital Light Microscopes

Bunton Instrument Co., Inc.

301-831-3434

www.buntgrp.com

Datacolor Inc.

800-982-6499

www.datacolor.com

Dunwell Tech

888-668-2442

http://dinolite.us/

Hirox-USA, Inc.

201-342-2600

www.hirox-usa.com

KEYENCE Corporation of America 888-539-3623

www.keyence.com

LECO Corporation

269-985-5496

www.leco.com

Microscope.com

540-904-0880

www.microscope.com

Microyn Technologies, Inc.

410-967-7118

www.microyntech.com

Motic Instruments, Inc.

604-303-9033

https://moticmicroscopes.com/

NanoAndMore USA, Inc.

843-521-1108

www.nanoandmore.com
Olympus

8322437900

https://www.olympus-ims. com/en/microscope/?utm

source=microscopy-today\&utm medium=marketing_network\&utm_ campaign=Dec_2020_I

E_All\&utm_content=listing

retroDIODE LLC

816-721-2517

www.retrodiode.com

Ted Pella, Inc.

800-237-3526

www.tedpella.com

Zarbeco, LLC

973-933-2043

www.zarbeco.com

\section{Dry Milling}

Clark-MXR, Inc.

734-426-2803

www.cmxr.com

Glen Mills Inc.

973-777-0777

www.GLENMILLS.com

\section{Dual Beam FIB/SEM}

Applied Beams LLC

503-608-7237

www.appliedbeams.com

FEI

$$
\text { 503-726-7500 }
$$

www.fei.com

ibss Group, Inc.

650-513-1488

www.ibssgroup.com

JC Nabity Lithography Systems

406-587-0848

www.jcnabity.com

Nanonics Imaging, Ltd.

866-220-6828

www.nanonics.co.il

Raith America, Inc.

631-738-9500

www.raith.com

Tescan

724-772-7433

www.tescan.com

TSS Microscopy

+15036164710

http://www.tssmicroscopy.com/

\section{DuraBeryllium Windows}

Moxtek, Inc.

801-225-0930

www.moxtek.com

\section{E Beam Lithography}

Electron Optica, Inc.

415-706-1557

www.electronoptica.com

HEIDENHAIN Corporation

847-490-1191

www.heidenhain.us
Integrated Dynamics Engineering IDE 781-326-5700

www.ideworld.com

JC Nabity Lithography Systems 406-587-0848

www.jcnabity.com

JEOL USA

978-535-5900

www.jeolusa.com

Raith America, Inc.

631-738-9500

www.raith.com

SAES Getters USA, Inc.

719-576-3200

www.saes-group.com

\section{EDS Detector Repairs}

\section{and Upgrades}

Advanced Analysis Technologies, LLC 608-798-2005

www.advancedanalysistech.com

Circuit Insights LLC

626-201-0488

www.LoopSlooth.com

IXRF, Inc.

512-386-6100

www.ixrfsystems.com

Oxford Instruments America, Inc. 800-447-4717

www.oxford-instruments.com

Quartz Imaging Corporation

604-488-3911

www.quartzimaging.com

RaySpec Ltd

+44 1628 5330-60

www.rayspec.co.uk

SII Nanotechnology USA, Inc.

www.siintusa.com

\section{EDS Detectors \& Systems}

Advanced Analysis Technologies, LLC 608-798-2005

www.advancedanalysistech.com

Bruker Corporation

608-276-3000

www.bruker.com

EDAX, Inc.

201-529-4880

www.edax.com

IXRF, Inc.

512-386-6100

www.ixrfsystems.com

Oxford Instruments

978-369-9933

www.oxinst.com

Oxford Instruments America, Inc.

800-447-4717

www.oxford-instruments.com

Oxford Instruments NanoAnalysis USA

+44 (0) 1494442255

https://nano.oxinst.com/ 
PNDetector $\mathrm{GmbH}$ +4989 309087-100 www.pndetector.de

PNSensor $\mathrm{GmbH}$ (49) 8930908713 www.pnsensor.de

Quartz Imaging Corporation 604-488-3911 www.quartzimaging.com

TREK, an Advanced Energy Company 1-800-FOR-TREK www.trekinc.com

\section{Education}

ATAGO U.S.A., Inc. 425-637-2107 http://www.atago-usa.com

Avian Rochester, LLC +1 585 259-5956 avianrochester.com

Berkeley Nucleonics Corp 1-415-453-9955 www.berkeleynucleonics.com

Cell Image Library (858) 534-0276 http://www.cellimagelibrary.org/home Hooke College of Applied Sciences 630-887-7100 www.hookecollege.com

International Centre for Diffraction Data (ICDD)

610-325-9814

www.icdd.com

Lehigh Microscopy School 610-758-1112 www.lehigh.edu/microscopy

McCrone Atlas of Microscopic Particles 630-887-7100 www.mccroneatlas.com

McCrone Research Institute 312-842-7100 www.mcri.org

Microscopy/Microscopy Education (MME) 972-924-5310 www.MicroscopyEducation.com

Royal Microscopical Society 01865265760 www.rms.org.uk

\section{Electrical Characterization}

Hysitron, a Bruker Company 952-835-6366 www.hysitron.com

Imina Technologies

Marktech Optoelectronics, Inc. (518) 956-2980 https://www.marktechopto.com

SmarAct Inc. 415-766-9006 www.smaract.com

\section{Electroformed Slits,}

Apertures and more

METRIGRAPHICS, LLC 800-261-2557

http://www.metrigraphicsllc.com/
Electron Backscatter

Diffraction (EBSD)

Bruker Corporation 608-276-3000 www.bruker.com

Bruker Nano Analytics 1-800-234-3028 www.bruker.com/nano-analysis EDAX, Inc. 201-529-4880 www.edax.com International Centre for Diffraction Data (ICDD)

610-325-9814

www.icdd.com

Oxford Instruments

978-369-9933

www.oxinst.com

NanoMEGAS 480-389-6816 www.nanomegasusa.com

Oxford Instruments 978-369-9933 www.oxinst.com

Oxford Instruments NanoAnalysis USA +44 (0) 1494442255

https://nano.oxinst.com/

Physical Electronics 952-828-6100 www.phi.com

Thermo Fisher Scientific (508) 479-6623 thermofisher.com/msr

\section{Electron Beam Joining System} Kimball Physics Inc.

603-878-1616

www.kimballphysics.com

Seron Technologies, Inc.

82-031-349-1411

www.serontech.co.kr

\section{Electron Crystallography \\ DECTRIS Ltd. \\ +41565002100 \\ www.dectris.com \\ NanoMEGAS \\ 480-389-6816 \\ www.nanomegasusa.com \\ X-Spectrum GmbH \\ +4915731711472 \\ https://x-spectrum.de/}

\section{Electron Energy Loss \\ Spectrometry (EELS)}

DECTRIS Ltd.

+41565002100

www.dectris.com

Gatan

925-463-0200

www.gatan.com

LeRoy Eyring Center for Solid State

Science

480-727-8578

https://le-csss.asu.edu

\section{Electron Guns}

Accuratus Corporation 908-213-7070

https://accuratus.com

Applied Physics Technologies, Inc. 503-434-5550 www.a-p-tech.com

Electron Optica, Inc. 415-706-1557 www.electronoptica.com

Hiden Analytical +44 (0) 1925445225 www.HidenAnalytical.com

Oregon Physics 503-601-0041 www.oregon-physics.com

Seron Technologies, Inc. 82-031-349-1411 www.serontech.co.kr

\section{Electron Microprobes/EPMA}

Advanced MicroBeam, Inc. 330-394-1255

www.advancedmicrobeam.com JEOL USA

978-535-5900

www.jeolusa.com

\section{Electron Microscopy Courses \\ Lehigh Microscopy School 610-758-1112 www.lehigh.edu/microscopy}

\section{Electron Probe}

Microanalyzers (EPMAs)

CAMECA

+33143346200

www.cameca.com

Oxford Instruments America, Inc. 800-447-4717 www.oxford-instruments.com

Seron Technologies, Inc. 82-031-349-1411

www.serontech.co.kr

Electrostatic Force Microscope TREK, an Advanced Energy Company 1-800-FOR-TREK www.trekinc.com

\section{Elemental Analysis \\ Verder Scientific Inc} (267) 757-0351 www.verder-scientific.com

\section{Embedding Materials}

Agar Scientific Ltd +44 (0)1279 215506 www.agarscientific.com

Electron Microscopy Sciences 215-412-8400 www.emsdiasum.com 


\section{A Guide to Microscopy Products}

Energy Beam Sciences, Inc.

(860) 653-0411

www.ebsciences.com

Ted Pella, Inc.

800-237-3526

www.tedpella.com

\section{EMI Cancellation}

Circuit Insights LLC

626-201-0488

www.LoopSlooth.com

ETS-Lindgren

630-307-7200

www.ets-lindgren.com

Field Management Services, Inc. 212-628-6860 x.205

www.fms-corp.com

Integrated Dynamics Engineering IDE 781-326-5700

www.ideworld.com

Spicer Consulting Limited $+44(0) 1234765773$

www.spicerconsulting.com

TMC

800-542-9725

www.techmfg.com

Vibration Engineering Consultants +1 831-465-9189

https://vibeng.com/

\section{Energy Dispersive X-ray}

Spectrometry (EDS)

Bruker Nano Analytics

1-800-234-3028

www.bruker.com/nano-analysis

EDAX, Inc.

201-529-4880

www.edax.com

MAX Detector Repair Group LLC 608-829-0110

maxdetector.com Micron Inc. 302-998-1184 https://microanalytical.com

Nion Co.

425-822-1521

www.nion.com

Oxford Instruments NanoAnalysis USA

+44 (0) 1494442255

https://nano.oxinst.com/

Quartz Imaging Corporation 604-488-3911

www.quartzimaging.com

PNDetector $\mathrm{GmbH}$ +4989 309087-100 www.pndetector.de

RaySpec Ltd

+44 1628 5330-60

www.rayspec.co.uk

Thermo Fisher Scientific

(508) 479-6623

thermofisher.com/msr
Energy Filters (EM)

Energy Beam Sciences, Inc.

(860) 653-0411

www.ebsciences.com

Gatan

925-463-0200

www.gatan.com

\section{Environmental Chambers}

Associated Environmental Systems 978.772.0022

www.AssociatedEnvironmental

Systems. com

AutoMate Scientific

510-845-6283

www.autom8.com

Bioptechs

724-282-7145

www.bioptechs.com

Electron Microscopy Sciences

215-412-8400

www.emsdiasum.com

Field Management Services, Inc. 212-628-6860 x. 205

www.fms-corp.com

TMC

800-542-9725

www.techmfg.com

\section{Environmental TEM}

Hummingbird Scientific 360-252-2737

www.hummingbirdscientific.com

LeRoy Eyring Center for Solid State Science

480-727-8578

https://le-csss.asu.edu

\section{Evaporation Sources}

Ted Pella, Inc. 800-237-3526

www.tedpella.com

\section{Evaporation Systems}

Denton Vacuum

856-439-9111

www.dentonvacuum.com

element $\mathrm{Pi}$

833-314-1593

www.elementpi.com

Quorum Technologies, Ltd.

$+44(0) 1323810981$

www.quorumtech.com

Facility Management System

Exprodo Software

562-275-1786

www.exprodo.com/calpendo

FOM Networks, Inc.

224-225-9168

www.fomnetworks.com
Failure Analysis

3D-Micromac AG

+49 371 40043-222

www.3d-micromac.com

Advanced MicroBeam, Inc. 330-394-1255

www.advancedmicrobeam.com

Advanced Research Corporation

www.arcnano.com Anasys Instruments 805-730-3310 www.

anasysinstruments.com

Anatech USA

775-657-8685

www.anatechusa.com

Anderson Materials Evaluation, Inc. 410-740-8562

www.andersonmaterials.com

Czitek

888.326 .8186

www.czitek.com

Cycle $\mathrm{GmbH}$

+49408998 89120

www.cyclelasers.com

Eurofins EAG Laboratories 800-366-3867

www.eag.com

Hysitron, Inc.

952-835-6366

www.hysitron.com

Kleindiek Nanotechnik

+4971213453950

www.kleindiek.com

Materials Evaluation and Engineering, Inc.

763-449-8870

mee-inc.com

McCrone Associates

630-887-7100

www.mccrone.com

Micron Inc.

302-998-1184

https://microanalytical.com

Polylnsight LLC www.polyinsight.com

Psylotech Inc.

+1-847-328-7100

www.psylotech.com

Quartz Imaging Corporation

604-488-3911

www.quartzimaging.com

Struers Inc.

440-871-0071

www.struers.com

Ted Pella, Inc. 800-237-3526

www.tedpella.com

Tescan

724-772-7433

www.tescan.com

Xradia, Inc.

925-701-3600

www.xradia.com

\section{FIB Accessories}

Advanced Research Corporation www.arcnano.com 


\section{A Guide to Microscopy Products}

Applied Beams LLC 503-608-7237

www.appliedbeams.com

E. A. Fischione Instruments, Inc. 724-325-5444

www.fischione.com

Evactron $₫$ by XEI Scientific, Inc. 650-369-0133

www.evactron.com

EXpressLO LLC

321-663-3806

www.EXpressLO.com

Hiden Analytical +44 (0) 1925445225

www. HidenAnalytical.com

Imina Technologies www.imina.ch

JC Nabity Lithography Systems 406-587-0848 www.jcnabity.com

Kammrath \& Weiss (516) 313-9742

https://www.kammrath-weiss.com/en/

L.A. Giannuzzi \& Associates LLC 321-663-3806 www.LAGiannuzzi.com

Nanonics Imaging, Ltd. 866-220-6828 www.nanonics.co.il

Oregon Physics 503-601-0041 www.oregon-physics.com

Protochips 919-377-0812 www.protochips.com

Raith America, Inc. 631-738-9500 www.raith.com

Struers Inc. 440-871-0071 www.struers.com

Ted Pella, Inc. 800-237-3526 www.tedpella.com

\section{FIB Courses}

Lehigh Microscopy School 610-758-1112 www.lehigh.edu/microscopy

\section{FIB Lift-out}

Agar Scientific Ltd +44 (0)1279 215506 www.agarscientific.com

Dune Sciences, Inc. 541-359-1959 www.dunesciences.com

EXpressLO LLC 321-663-3806 www.EXpressLO.com

Graticules Optics Ltd +441732360460 www.graticulesoptics.com

Kleindiek Nanotechnik +4971213453950 www.kleindiek.com
L.A. Giannuzzi \& Associates LLC 321-663-3806 www.LAGiannuzzi.com

MEO Engineering Company +1-978-305-0479 www.fibsemproducts.com

Ted Pella, Inc.

800-237-3526

www.tedpella.com

Tescan

724-772-7433

www.tescan.com

\section{FIB-SEMs}

Applied Beams LLC 503-608-7237 www.appliedbeams.com

Applied Physics Technologies, Inc. 503-434-5550 www.a-p-tech.com

FEI

503-726-7500

www.fei.com

Tescan

724-772-7433

www.tescan.com

ZEISS

1-800-233-2343

www.zeiss.com/microscopy

FIB Service Laboratories

Applied Beams LLC

503-608-7237

www.appliedbeams.com

Semion

503-332-9668

www.semionco.com

TSS Microscopy

+1503616 4710

http://www.tssmicroscopy.com/

Fiber Optic Illuminators

LASERGLOW Technologies

+1416-729-7976

www.laserglow.com

\section{Field Emission Sources}

\section{(See Filaments)}

\section{Filaments and Filament}

\section{Rebuilding}

Agar Scientific Ltd +44 (0)1279215506 www.agarscientific.com

Applied Beams LLC 503-608-7237

www.appliedbeams.com

Applied Physics Technologies, Inc. 503-434-5550 www.a-p-tech.com

Energy Beam Sciences, Inc. (860) 653-0411 www.ebsciences.com

Kimball Physics Inc. 603-878-1616 www.kimballphysics.com
Ted Pella, Inc. 800-237-3526 www.tedpella.com

Field Microscope

ioLight Magnificent Mobile Microscopes +44 (0)333202 7101

ioLight.co.uk

\section{Filters (See Optical Filters)}

\section{Fixatives and Chemicals}

General Data Healthcare 844-643-1129 www.general-data.com

HTA Photomask 408 452-5500 www.htaphotomask.com

Ladd Research 802-658-4961 www.laddresearch.com

Ted Pella, Inc. 800-237-3526 www.tedpella.com

Tousimis 301-881-2450

www.tousimis.com

\section{Fluorescence-Lifetime} Imaging Microscopy (FLIM) Lambert Instruments BV +31 (0) 505018461 www.lambertinstruments.com

\section{Fluorescence Microscopy}

Alluxa, Inc.

855-4Alluxa

www.alluxa.com

A.P.E Angewandte Physik \& Elektronik $\mathrm{GmbH}$

+4930 986 011-30

www.ape-berlin.de

Applied Precision, aGE Healthcare

Company

425-829-9439

ARGOLIGHT

+33 (0)564 310850

www.argolight.com

Armadillo Sia

(408) 834-7422

https://armadillosia.com

Axiom Optics

617-221-6636

www.axiomoptics.com

BioTek Instruments, Inc.

888-451-5171

www.biotek.com

Boston Electronics

617-566-3821

www.boselec.com 
Bunton Instrument Co., Inc. 301-831-3434 www.buntgrp.com

Bruker AFM Probes 520-741-1044 www.brukerafmprobes.com

Bruker Nano Surfaces and Metrology 520-741-1044 ext.1075 www.bruker.com/nano

Chroma Technology Corp. 800-824-7662 www.chroma.com

CRAIC Technologies, Inc. 310-573-8180 www.microspectra.com

\section{CoolLED}

+44 (0)1264 323040

www.coolled.com

CrestOptics www.crestopt.com

DELMIC BV +31(0)157440158 www.delmic.com

DiCon Fiberoptics, Inc. 510-620-5167 www.diconfiberoptics.com

Dunwell Tech 888-668-2442 http://dinolite.us/

Etaluma, Inc.

760-298-2355

www.etaluma.com

Euresys Inc +19497430612 www.euresys.com

Forth Dimension Displays Ltd. +44 0 1383-827-950 www.forthdd.com

Goldstone Scientific LLC +1 248-424-9390 www.GoldstoneScientific.com Holo/Or Ltd. +972-8-9409687 www.holoor.co.il/

HORIBA Scientific 732-494-8660 www.horiba.com/scientific

ibidi $\mathrm{GmbH}$ +4989520 46 17-0 www.ibidi.com

Imaging and Analysis, LLC 651-788-2261 www.imagingandanalysis.com

KEYENCE Corporation of America 888-539-3623 www.keyence.com

Kimmon Koha USA, Inc. (303) 754-0401 www.kimmon.com

KMLabs, Inc. 303-544-9068 www.kmlabs.com

LASERGLOW Technologies +1416-729-7976 www.laserglow.com

Leica Microsystems 800-248-0123 www.leica-microsystems.com
Lumencor

503-213-4269

www.lumencor.com

LW Scientific

800-726-73 45

www.Iwscientific.com

Mad City Labs, Inc. 608-298-0855

www.madcitylabs.com

Meadowlark Optics 303-833-4333

www.meadowlark.com

Media Cybernetics

301-495-3305

www.mediacy.com

MICROS Produktions-und HandelsgmbH www.micros.at

Microscopy/Microscopy Education (MME) 972-924-5310

www.MicroscopyEducation.com

Mightex Systems

1-925-218-1885

www.mightexsystems.com

Miltenyi Biotec B.V. \& Co. KG +49220483060

www.miltenyibiotec.com

Motic Instruments, Inc. 604-303-9033 https://moticmicroscopes.com/

NanoAndMore USA, Inc.

843-521-1108

www.nanoandmore.com

NIGHTSEA

781-791-9508

www.nightsea.com

Olympus Life Science (781) 419-3687

www.olympus-lifescience.com

Omega Optical, LLC

802-251-7300

www.omegafilters.com

OSI Optoelectronics Inc.

+1 310-355-2812

www.osioptoelectronics.com

PCO-TECH Inc.

+1 (248) 2768820

www.pco-tech.com

Phase Focus Ltd

+441142866377

www.phasefocus.com

PicoQuant GmbH

+49-(0)30-6392-6929

www.picoquant.com

Prizmatix

1 248-436-8085

www.prizmatix.com

Quantum Composers Inc 406-582-0227

Quantumcomposers.com

retroDIODE LLC

816-721-2517

www.retrodiode.com

Scientific Volume Imaging

+31356421626

www.svi.nl
Spectrolight Inc. 949-800-7780

www.spectrolightinc.com

Stanford Photonics, Inc. 650-969-5991 www.stanfordphotonics.com

Sutter Instrument 415-883-0128 www.sutter.com

TechnoSpex Pte Ltd +6562766928 www.technospex.com

Teledyne Photometrics 520-889-9933 photometrics.com

TOFRA, Inc. 650-387-5497 www.tofrainc.net

UQG (Lab Glasses) + 44 (0) 1223420329 www.uqgoptics.com

US Fiberoptec Technology Inc. 1 (408) 834-7422 usfiberoptec.com

Used-Line 845-287-0066 Used-Line.com

Ushio America, Inc. 800-838-7446 www.ushio.com

UVP LLC

909-946-3197

www.uvp.com

Vee Gee Scientific 800-423-8842 www.veegee.com

Vutara

801-512-2664

www.vutara.com

Zarbeco, LLC 973-933-2043 www.zarbeco.com

Fluorescence Resonance Energy Transfer (FRET)

Alluxa, Inc. 855-4Alluxa www.alluxa.com

Axiom Optics 617-221-6636 www.axiomoptics.com

Boston Electronics 617-566-3821 www.boselec.com

FEl Munich $\mathrm{GmbH}$ +4989895662 0 www.fei.com

Lambert Instruments +31 (0) 505018461 www.lambertinstruments.com

LASERGLOW Technologies +1416-729-7976 www.laserglow.com

Photometrics 520-889-9933 www.photometrics.com 


\section{A Guide to Microscopy Products}

\section{Fluorophores}

Miltenyi Biotec B.V. \& Co. KG $+49220483060$

www.miltenyibiotec.com

\section{Forster Resonance Energy \\ Transfer (FRET) \\ LASERGLOW Technologies $+1416-729-7976$ \\ www.laserglow.com}

\section{Focused Ion Beam \\ Systems/ Workstations \\ FEl}

503-726-7500

www.fei.com

Hitachi High Technologies America, Inc. 800-253-3053

www.hitachi-hightech.com/us

TSS Microscopy

+15036164710

http://www.tssmicroscopy.com/

\section{Forensic Testing Supplies}

Spectronics Corporation

516-333-4840

www.spectroline.com

Ted Pella, Inc.

800-237-3526

www.tedpella.com

\section{Freeze Drying Equipment}

Linkam Scientific Instruments +44 (0) 1737363476

www.linkam.co.uk

McCrone Microscopes \& Accessories 630-887-7100

www.mccrone.com

Quorum Technologies, Ltd.

$+44(0) 1323810981$

www.quorumtech.com

\section{Friction Analyzer \\ Kett US}

www.kett.com

\section{FT-IR Microscopy}

Anasys Instruments

805-730-3310

www.anasysinstruments.com

Armadillo Sia

(408) 834-7422

https://armadillosia.com

BioTools

561-625-0133

www.btools.com

Bruker Optics

978-663-3660

www.bruker.com

CRAIC Technologies, Inc.

310-573-8180

www.microspectra.com
CRYO Industries of America, Inc.

603-621-9957

www.cryoindustries.com

Czitek

888.326.8186

www.czitek.com

HelioWorks, Inc.

707-578-7200

www.helioworks.com

Instec, Inc.

303-444-4608

www.instec.com

Janis Research Company, LLC

781-491-0888

www.janis.com

Lake Shore Cryotronics

781-491-0888

www.lakeshore.com/janis

McCrone Associates

630-887-7100

www.mccrone.com

Microscopy/Microscopy Education (MME)

972-924-5310

www.MicroscopyEducation.com

MicroSpec On-site

972-924-5310

microspec-onsite.com

MKS Instruments, Inc.

978-645-5500

www.mksinst.com

neaspec $\mathrm{GmbH}$

49-176-2320-3996

www.neaspec.com

RedWave Technology

203-628-4833

www.redwavetech.com

SENSORS UNLIMITED INC., A PART OF

COLLINS AEROSPACE

609-333-8200

www.sensorsinc.com

S.T.Japan-Europe $\mathrm{GmbH}$

+49 (0) 2234-956372

www.stjapan.de

UQG (Lab Glasses)

+ 44 (0) 1223420329

www.uqgoptics.com

US Fiberoptec Technology Inc.

1 (408) 834-7422

usfiberoptec.com

\section{Furnaces}

Glen Mills Inc.

973-777-0777

www.GLENMILLS.com

Verder Scientific Inc

(267) 757-0351

www.verder-scientific.com

\section{Gauges (Vacuum)}

BrandTech Scientific, Inc.

888-522-2726

www.brandtech.com

Kurt J. Lesker Company

412-387-9200

www.lesker.com
MKS Instruments, Inc.

978-645-5500

www.mksinst.com

Nor-Cal Products by Pfeiffer Vacuum 800-824-4166

www.n-c.com

Pfeiffer Vacuum

603-578-6500

www.pfeiffer-vacuum.com

Ted Pella, Inc.

800-237-3526

www.tedpella.com

\section{Glass Knives}

Boeckeler Instruments, Inc. 520-745-0001

www.boeckeler.com

Ted Pella, Inc.

800-237-3526

www.tedpella.com

\section{Gloves}

Ted Pella, Inc.

800-237-3526

www.tedpella.com

\section{Glow Discharge Cleaners}

Ted Pella, Inc.

800-237-3526

www.tedpella.com

\section{Gold Nanoparticles/Probes}

Nanoprobes, Inc.

1-877-447-6266, (631) 205-9490

www.nanoprobes.com

Ted Pella, Inc.

800-237-3526

www.tedpella.com

Grids (EM)

Accuratus Corporation 908-213-7070

https://accuratus.com

Electron Microscopy Sciences 215-412-8400

www.emsdiasum.com

Graticules Optics Ltd

+441732360460

www.graticulesoptics.com

MiTeGen

1-607-266-8877

www.mitegen.com

Ted Pella, Inc.

800-237-35 26

www.tedpella.com

TEMWindows

585-214-0585

www.TEMWindows.com

YW MEMS

+86-512-6726-6051

http://www.cleansin.com/ 


\section{A Guide to Microscopy Products}

\author{
Hall Effect Measurement \\ System (HEMS) \\ NanoMagnetics Instruments Ltd. \\ +441865522989 \\ www.nanomagnetics-inst.com
}

\author{
Heating Stages \\ AutoMate Scientific \\ 510-845-6283 \\ www.autom8.com \\ Bioscience Tools \\ 1-877-853-9755 \\ www.biosciencetools.com \\ Boston Electronics \\ 617-566-3821 \\ www.boselec.com \\ Gatan \\ 925-463-0200 \\ www.gatan.com \\ ibidi $\mathrm{GmbH}$ \\ +4989520 46 17-0 \\ www.ibidi.com \\ Instec, Inc. \\ 303-444-4608 \\ www.instec.com \\ Kammrath \& Weiss \\ (516) 313-9742 \\ https://www.kammrath-weiss.com/en/ \\ Linkam Scientific Instruments \\ +44 (0) 1737363476 \\ www.linkam.co.uk \\ McCrone Microscopes \& Accessories \\ 630-887-7100 \\ www.mccrone.com \\ Psylotech Inc. \\ +1-847-328-7100 \\ www.psylotech.com
}

\section{Helium \& Ion Microscopes}

JC Nabity Lithography Systems

406-587-0848

www.jcnabity.com

ZEISS

1-800-233-2343

www.zeiss.com/microscopy

\section{High Definition Cameras}

Basler AG

+49-4102-463-500 www.baslerweb. com

CANON MEDICAL, Video Sensing

Division

810-357-5022

https://us.medical.canon/VSD

Dage-MTI

219-872-5514

www.dagemti.com

Martin Microscope Company

864-242-3424

www.MartinMicroscope.com

TRU-Vu Monitors, Inc.

847-259-2344

www.TRU-VuMonitors.com
High Purity Metals,

Alloys and Polymers

Advent Research Materials Ltd +44 (0) 1865884440

www.advent-rm.com

High Throughput Imaging

Applications

Thermo Fisher Scientific

(508) 479-6623

thermofisher.com $/ \mathrm{msr}$

High Speed Imaging

Advent Research Materials Ltd +44 (0) 1865884440 www.advent-rm.com

DECTRIS Ltd.

+41565002100

www.dectris.com

Euresys Inc

+19497430612

www.euresys.com

Focus e-Beam Technology (Beijing)

Co.,Ltd.

86-10-67832505

www.focus-ebeam.com

Lambert Instruments

+31 (0) 505018461

www.lambertinstruments.com

Lumencor

503-213-4269

www.lumencor.com

Navitar

585-359-4000

www.navitar.com

NTT Advanced Technology Corporation www.ntt-at.com

PCO-TECH Inc.

+1 (248) 2768820

www.pco-tech.com

Pembroke Instruments, LLC

415-860-4127

https://pembrokeinstruments.com

Phaseview

+33954030543

www.phaseview.com

Photron

858-684-3555

www.photron.com

Pixelink

1-833-247-1211

www.pixelink.com

Princeton Infrared Technologies, Inc. 609-917-3380

www.princetonirtech.com

SCHOTT North America, Inc. Lighting and Imaging

+1-508-765-9744

www.us.schott.com/lightingimaging

SENSORS UNLIMITED INC., A PART OF

COLLINS AEROSPACE

609-333-8200

www.sensorsinc.com

Tescan

724-772-7433

www.tescan.com
X-Spectrum GmbH +4915731711472

https://x-spectrum.de/

YXLON / Comet Technologies USA 877-972-9100

www.yxlon.com

High Speed sCMOS Sensors

Advanced Microscopy Techniques 978-774-5550

www.amtimaging.com

\section{Histology Supplies}

General Data Healthcare

844-643-1129

www.general-data.com

Globe Scientific Inc.

www.globescientific.com

Ted Pella, Inc

800-237-3526

www.tedpella.com

\section{Histology Stains}

General Data Healthcare

844-643-1129

www.general-data.com

Hoffman Modulation Microscopy

Microscopy/Microscopy Education (MME) 972-924-5310

www.MicroscopyEducation.com

Navitar

585-359-4000

www.navitar.com

Hot Plates

Instec, Inc.

303-444-4608 www.instec.com

PRO Scientific

(203) 267-4600

www.proscientific.com

Ted Pella, Inc.

800-237-3526

www.tedpella.com

\section{Illuminators}

Armadillo Sia

(408) 834-7422

https://armadillosia.com

BLC America

714-889-4116

www.blc-america.com

BOCK OPTRONICS INC.

416-674-2804

www.bockoptronics.ca

Bulbworks, Inc.

973-584-7171

www.bulbworks.com

Bunton Instrument Co., Inc.

301-831-3434

www.buntgrp.com

Cadmet Inc

800-543-7282

www.cadmet.com

CeramOptec Industries, Inc. 413-525-0600

www.ceramoptec.com 


\section{A Guide to Microscopy Products}

CoolLED Limited

1-800-877-0128

www.coolled.com

CytoViva, Inc.

1-334-321-3901

www.cytoviva.com

Edge-3d

808.344 .5954

www.edge-3d.com

Energetiq Technology

781-939-0763

www.energetiq.com

Gamma Scientific

858-279-8034

www.gamma-sci.com

Goldstone Scientific LLC

+1 248-424-9390

www.GoldstoneScientific.com

JENOPTIK Optical Systems

561-881-7400

www.jenoptik.com

LASERGLOW Technologies

+1416-729-7976

www.laserglow.com

Mightex Systems

1-925-218-1885

www.mightexsystems.com

Nanodyne Measurement Systems, Inc.

651-323-8592

http://www.nano-dyne.com

Opto Diode Corporation, An ITW

Company

805-499-0335

Power Technology, Inc.

501.407.0712

www.powertechnology.com

Prizmatix

$1248-436-8085$

www.prizmatix.com

SCHOTT North America, Inc. Lighting and

Imaging

+1-508-765-9744

www.us.schott.com/lightingimaging

Spectrolight Inc.

949-800-7780

www.spectrolightinc.com

retroDIODE LLC

816-721-2517

www.retrodiode.com

Ted Pella, Inc.

800-237-3526

www.tedpella.com

TOFRA, Inc.

650-387-5497

www.tofrainc.net/

US Fiberoptec Technology Inc.

1 (408) 834-7422

usfiberoptec.com

Ushio America, Inc.

800-838-7446

www.ushio.com

Image Analysis and Processing

Adsys Controls, Inc.

949-682-5430

www.AdsysControls.com
Applied Electro-Optics, Inc.

512-686-0752

www.aeousa.com

Applied Precision, aGE Healthcare

Company

425-829-9439

www.gelifesciences.com

BioTek Instruments, Inc.

888-451-5171

www.biotek.com

Black Mesa Imaging, LLC

484-693-0563

www.blackmesa-imaging.com

Buehler

www.buehler.com

CytoViva, Inc.

1-334-321-3901

www.cytoviva.com

Datacolor Inc.

800-982-6499

www.datacolor.com

DiCon Fiberoptics, Inc.

510-620-5167

www.diconfiberoptics.com

Diffraction Limited

+1-613-225-2732

https://www.diffractionlimited.com

Edge-3d

808.344.5954

www.edge-3d.com

EMSIS GmbH

+49251297962 0

www.emsis.eu

Euresys Inc

+19497430612

www.euresys.com

FEI

503-726-7500

www.fei.com

Fluid Imaging Technologies, Inc.

207-846-6100

www.fluidimaging.com

GIGAmacro

415-841-3322

www.gigamacro.com

HEIDENHAIN Corporation

847-490-1191

www.heidenhain.us

HREM Research, Inc.

81-493-35-3919

www.hremresearch.com

Image Metrology A/S

+4546923400

www.imagemet.com

Imaging and Analysis, LLC

651-788-2261

www.imagingandanalysis.com

Lumenera Corporation

613-736-4077

www.lumenera.com

Malvern Panalytical

+1 800 279-7297

www.malvernpanalytical.com

Media Cybernetics

301-495-3305

www.mediacy.com
Micro Photonics

610-366-7103

www.microphotonics.com

Microscopy/Microscopy Education (MME) 972-924-5310

www.MicroscopyEducation.com

NanoMEGAS

480-389-6816

www.nanomegasusa.com

Object Research Systems

514-843-3861

www.theobjects.com

Particle Technology Labs

630-969-2703

www.particletechlabs.com

Phase Focus Ltd

+44114286 6377

www.phasefocus.com

PicoQuant $\mathrm{GmbH}$

+49-(0)30-639 2-6929

www.picoquant.com

Photometrics

520-889-9933

www.photometrics.com

Photron

858-684-3555

www.photron.com

PNDetector $\mathrm{GmbH}$

+49 89 309087-100

www.pndetector.de

Quartz Imaging Corporation

604-488-3911

www.quartzimaging.com

ResAlta Research Technologies Corp.

303-748-43 46

www.resaltatech.com

Scientific Instruments \& Applications, Inc. 770-232-7785

www.sia-cam.com

Scientific Volume Imaging

+31356421626

www.svi.nl

SENSORS UNLIMITED INC., A PART OF COLLINS AEROSPACE

609-333-8200

www.sensorsinc.com

Seron Technologies, Inc.

82-031-349-1411

www.serontech.co.kr

Stanford Photonics, Inc.

650-969-5991

www.stanfordphotonics.com

Tescan

724-772-7433

www.tescan.com

TVIPS GmbH

49-89-8506567

www.tvips.com

Visage Imaging

858-356-5517

www.visageimaging.com

Wolfram Research, Inc.

217-398-0700

https://www.wolfram.com

Xradia, Inc.

925-701-3600

www.xradia.com 
Image Analysis Software

Adsys Controls, Inc. 949-682-5430 www.AdsysControls.com

APPLIED IMAGE Inc. 585-482-0300 www.appliedimage.com

Basler AG +49-4102-463-500 www.baslerweb.com

Black Mesa Imaging, LLC 484-693-0563 www.blackmesa-imaging.com BOCK OPTRONICS INC. 416-674-2804 www.bockoptronics.ca DigiSENS www.digisens3d.com

Digital Surf 0033381504800 www.digitalsurf.com

Diffraction Limited +1-613-225-2732 https://www. diffractionlimited.com

Edge-3d 808.344.5954 www.edge-3d.com

Euresys Inc +19497430612 www.euresys.com

HREM Research, Inc. 81-493-35-3919 www.hremresearch.com Image Metrology A/S +454692 3400 www.imagemet.com

Media Cybernetics 301-495-3305 www.mediacy.com

Photron 858-684-3555 www.photron.com

Scientific Volume Imaging +31356421626 www.svi.nl

SPOT Imaging Solutions 586-731-6000 www.spotimaging.com

Wolfram Research, Inc. 217-398-0700 https://www.wolfram.com ZEISS

1-800-233-2343

www.zeiss.com/microscopy

Impedance Spectrosocpy

Applied BioPhysics

518-880-6860

www.biophysics.com

Immunolabeling

DiCon Fiberoptics, Inc. 510-620-5167 www.diconfiberoptics.com
Nanoprobes, Inc. 1-877-447-6266, (631) 205-9490 www.nanoprobes.com

Ted Pella, Inc. 800-237-35 26 www.tedpella.com

Incubators for Live Cell Imaging

Applied BioPhysics 518-880-6860 www.biophysics.com

Bioptechs

724-282-7145

www.bioptechs.com

Bioscience Tools

1-877-853-9755

www.biosciencetools.com

BioTek Instruments, Inc.

888-451-5171

www.biotek.com

ibidi $\mathrm{GmbH}$

+498952046 17-0

www.ibidi.com

\section{Indenters}

Bruker Nano Surfaces and Metrology 520-741-1044 ext.1075 www.bruker.com/nano

Micro Star Technologies 800-533-2509

www.microstartech.com

Infra-Red Microscopes

Anasys Instruments 805-730-3310 www.anasysinstruments.com

Applied Electro-Optics, Inc. 512-686-0752 www.aeousa.com

BioTools

561-625-0133

www.btools.com

Bruker Nano Surfaces and Metrology 520-741-1044 ext. 1075 www.bruker.com/nano

Bruker Optics 978-663-3660 www.bruker.com

Czitek

888.326.8186

www.czitek.com

HelioWorks, Inc. 707-578-7200 www.helioworks.com

PIKE Technologies, Inc. 608-271-2721 www.piketech.com

Princeton Infrared Technologies, Inc. 609-917-3380 www.princetonirtech.com

RedWave Technology 203-628-4833 www.redwavetech.com

Zarbeco, LLC 973-933-2043 www.zarbeco.com
In-Situ Atomic Force

Microscopes (AFMs)

Angstrom Scientific, Inc. 201-962-7222

www.angstrom.us

\section{In-Situ SEM}

Bruker Nano Surfaces and Metrology 520-741-1044 ext. 1075 www.bruker.com/nano

Gatan

925-463-0200

www.gatan.com

Hysitron, a Bruker Company 952-835-6366 www.hysitron.com

Kammrath \& Weiss (516) 313-9742 https://www.kammrath-weiss.com/en/

Materials Analysis Technology Inc. +866-3-611-6678 www.bioma-tek.com

NenoVision +420605287732 www.nenovision.com

NORCADA INC.

780-431-9637 www.norcada.com

Psylotech Inc. +1-847-328-7100 www.psylotech.com

Quantum Design, Inc. 858-481-4400 https://www.qdusa.com/ YW MEMS

+86-512-6726-6051

http://www.cleansin.com/

\section{In-Situ TEM}

Advanced Microscopy Techniques 978-774-5550 www.amtimaging.com

Bruker Nano Surfaces and Metrology 520-741-1044 ext. 1075 www.bruker.com/nano

DECTRIS Ltd. +41565002100 www.dectris.com

Direct Electron, LP +1 858-384-0291 www.directelectron.com FEI 503-726-7500 www.fei.com

Gatan

925-463-0200 www.gatan.com

Hitachi High Technologies America, Inc. 800-253-3053 www.hitachi-hightech.com/us

Hysitron, a Bruker Company 952-835-6366 www.hysitron.com 


\section{A Guide to Microscopy Products}

Materials Analysis Technology Inc. +866-3-611-6678

www.bioma-tek.com

NORCADA INC.

780-431-9637

www.norcada.com

Protochips

919-377-0812

www.protochips.com

YW MEMS

+86-512-6726-6051

http://www.cleansin.com/

\section{Integrated EDS, EBSD, \\ WDS Microanalysis \\ Oxford Instruments \\ 978-369-9933 \\ www.oxinst.com \\ Tescan \\ 724-772-7433 \\ www.tescan.com}

\section{Inverted Light Microscopes}

Etaluma, Inc.

760-298-2355

www.etaluma.com

ioLight Magnificent Mobile Microscopes +44 (0)3332027101

ioLight.co.uk

LW Scientific

800-726-7345

www.Iwscientific.com

nPoint, Inc.

608-824-1770

www.npoint.com

Ted Pella, Inc.

800-237-3526

www.tedpella.com

\section{Ion Beam Milling}

COXEM CO., LTD

+82-42-861-1686

www.coxem.com

E. A. Fischione Instruments, Inc. 724-325-5444

www.fischione.com

FEI

503-726-7500

www.fei.com

Gatan

925-463-0200

www.gatan.com

Hitachi High Technologies America, Inc. 800-253-3053

www.hitachi-hightech.com/us

JEOL USA

978-535-5900

www.jeolusa.com

Materials Evaluation and Engineering, Inc.

763-449-8870

mee-inc.com

Norsam Technologies, Inc.

505-984-1133

www.norsam.com
Oregon Physics

503-601-0041

www.oregon-physics.com

Siskiyou Corporation

541-479-8697

www.siskiyou.com

Tescan

724-772-7433

www.tescan.com

\section{Ion Beam Polishers}

Fischone Instruments

724-325-5444

www.fischone.com

Gatan

925-463-0200

www.gatan.com

\section{Ion Guns}

Accuratus Corporation 908-213-7070

https://accuratus.com

Kimball Physics Inc.

603-878-1616

www.kimballphysics.com

Oregon Physics

503-601-0041

www.oregon-physics.com

\section{Ion Microscopes}

Applied Beams LLC

503-608-7237

www.appliedbeams.com

Ion Pumps, New and Rebuilding

Duniway Stockroom Corp.

650-969-8811

www.duniway.com

Gamma Vacuum

952-445-4841

www.gammavacuum.com

\section{Journals}

International Centre for Diffraction Data (ICDD)

610-325-9814

www.icdd.com

McCrone Research Institute

312-842-7100

www.mcri.org

McCrone Research Institute, Chicago

312-842-7100

www.mcri.org

Microscopy and Microanalysis 212-337-5900

www.joumals.cambridge.org/MAM

Modem Microscopy

630-887-7100

www.modernmicroscopy.com

Royal Microscopical Society

01865265760

www.rms.org.uk

\section{Knifemakers}

Delaware Diamond Knives, Inc. 800-222-5143

www.ddk.com

Microtome Service Company 315-451-1404

www.microtomeserviceco.com

Micro Star Technologies

800-533-2509

www.microstartech.com

RMC Boeckeler

1-520-745-0001

www.rmcboeckeler.com

Ted Pella, Inc.

800-237-3526

www.tedpella.com

\section{Knife Resharpening}

DiATOME U.S.

2154128390

www.emsdiasum.com

\section{Knives}

Delaware Diamond Knives, Inc. 800-222-5143

www.ddk.com

DiATOME U.S.

2154128390

www.emsdiasum.com

Micro Star Technologies

800-533-2509

www.microstartech.com

S.T.Japan-Europe $\mathrm{GmbH}$ +49 (0) 2234-956372

www.stjapan.de

Ted Pella, Inc.

800-237-3526

www.tedpella.com

B6 Sources (See Filaments)

Laboratory Fume Hoods

Energy Beam Sciences, Inc.

(860) 653-0411

www.ebsciences.com

General Data Healthcare

844-643-1129

www.general-data.com

HEMCO Corporation

18007794362

www.HEMCOcorp.com

Kewaunee Scientific Corporation 7048737202

www.kewaunee.com

\section{Laboratory Furniture}

HEMCO Corporation

18007794362

www.HEMCOcorp.com

Kewaunee Scientific Corporation 7048737202

www.kewaunee.com 


\section{A Guide to Microscopy Products}

Laboratory Information

Management System (LIMS)

Agilent Crosslab, iLab Operations

Software

1-617-297-2805/1-800-690-2957

www.agilent.com/chem/ilab

Exprodo Software

562-275-1786

www.exprodo.com/calpendo

FOM Networks, Inc.

224-225-9168

www.fomnetworks.com

\section{Laboratory Safety Supplies}

Bel-Art Products www.belart.com

HEMCO Corporation

18007794362

www.HEMCOcorp.com

Nextteq LLC

877-312-2333

www.nextteq.com

Ted Pella, Inc.

800-237-3526

www.tedpella.com

\section{Laminar Flow Hoods \\ HEMCO Corporation 18007794362 \\ www.HEMCOcorp.com \\ Kewaunee Scientific Corporation 7048737202 \\ www.kewaunee.com}

\section{Lamps and Light Sources}

Accuratus Corporation 908-213-7070 https://accuratus.com

BLC America

714-889-4116

www.blc-america.com

Bulbworks, Inc.

973-584-7171

www.bulbworks.com

Cadmet Inc

800-543-7282

www.cadmet.com

CoolLED

$+44(0) 1264323040$

www.coolled.com

Cycle $\mathrm{GmbH}$

+49408998 89120

www.cyclelasers.com

Energetiq Technology

781-939-0763

www.energetiq.com

Gamma Scientific

858-279-8034

www.gamma-sci.com

Goldstone Scientific LLC

+1 248-424-9390

www.GoldstoneScientific.com
Hamamatsu Corporation 908-231-0960

www.hamamatsu.com

Laser 2000 (UK) Ltd.

+44 (0) 01933461666

www.laser2000.co.uk

Laser Components USA, Inc. (603) 8217040

www.lasercomponents.com

Lumencor

503-213-4269

www.lumencor.com

Marktech Optoelectronics, Inc. (518) 956-2980

https://www.marktechopto.com

Prizmatix

$1248-436-8085$

www.prizmatix.com

retroDIODE LLC

816-721-2517

www.retrodiode.com

SCHOTT North America, Inc. Lighting and Imaging

+1-508-765-9744

www.us.schott.com/lightingimaging

Spectronics Corporation

516-333-4840

www.spectroline.com

Sutter Instrument

415-883-0128

www.sutter.com

Ushio America, Inc.

800-838-7446

www.ushio.com

UVP LLC

909-946-3197

www.uvp.com

\section{Large Format}

\section{Mpix+ TEM Imaging}

Advanced Microscopy Techniques

978-774-5550

www.amtimaging.com

\section{Lasers}

3D-Micromac AG

+49371 40043-222

www.3d-micromac.com

CeramOptec Industries, Inc. 413-525-0600 www.ceramoptec.com

Clark-MXR, Inc.

734-426-2803

www.cmxr.com

CoboltAB

$+4-685-459-1230$

www.cobolt.se

Cycle $\mathrm{GmbH}$ +49408998 89120 www.cyclelasers.com

DayOptics, Inc. +8659183215681

www.dayoptics.com

Kimmon Koha USA, Inc.

(303) 754-0401

www.kimmon.com
KMLabs, Inc.

303-544-9068

www.kmlabs.com

Laser 2000 (UK) Ltd. +44 (0) 01933461666

www.laser2000.co.uk

LASERGLOW Technologies

+1416-729-7976

www.laserglow.com

Pantec Engineering AG

+423377 1333

www.pantec-medicallaser.com

PicoQuant GmbH

+49-(0)30-6392-6929

www.picoquant.com

Power Technology, Inc.

501.407.0712

www.powertechnology.com

RPMC Lasers, Inc.

(636) 272-7227

https://www.rpmclasers.com

Quantum Composers Inc

406-582-0227

Quantumcomposers.com

VEROPTICS

1-800-939-6627

www.veroptics.com

\section{Laser Scanning Confocal \\ Microscopes}

Abberior Instruments America LLC

+15619729300

www.abberior-instruments-america.com

Axiom Optics

617-221-6636

www.axiomoptics.com

Boston Electronics

617-566-3821

www.boselec.com

KEYENCE Corporation of America

888-539-3623

www.keyence.com

NTT Advanced Technology Corporation www.ntt-at.com

Olympus

8322437900

https://www.olympus-ims.

com/en/microscope/?utm

source=microscopy-today\&utm

medium=marketing_network\&utm

campaign=Dec_2020_IE_All\&utm_

content=listing

Olympus Life Science

781) 419-3687

www.olympus-lifescience.com

\section{Leak Detection}

MKS Instruments, Inc.

978-645-5500

www.mksinst.com

Nextteq LLC

877-312-2333

www.nextteq.com 
Spectronics Corporation 516-333-4840

www.spectroline.com

TGS Technologies, LLC

724-453-3865

www.tgstechnologies.net

\section{Leak Detectors}

Pfeiffer Vacuum 603-578-6500

www.pfeiffer-vacuum.com

ULVAC Technologies, Inc.

978-686-7550

www.ulvac.com

\section{LED Illumination}

Alluxa, Inc.

855-4Alluxa

www.alluxa.com

Alrad Imaging +44 (0)1635 30345

www.alrad.com

BLC America

714-889-4116

www.blc-america.com

Bulbworks, Inc.

973-584-7171

www.bulbworks.com

Cadmet Inc

800-543-7282

www.cadmet.com

Gamma Scientific

858-279-8034

www.gamma-sci.com

Goldstone Scientific LLC

+1 248-424-9390

www.GoldstoneScientific.com

CoolLED

$+44(0) 1264323040$

www.coolled.com

Lumencor

503-213-4269

www.lumencor.com

Marktech Optoelectronics, Inc.

(518) 956-2980

https://www.marktechopto.com

Mightex Systems

1-925-218-1885

www.mightexsystems.com

Nanodyne Measurement Systems, Inc.

651-323-8592

http://www.nano-dyne.com

NIGHTSEA

781-791-9508

www.nightsea.com

Opto Diode Corporation, An ITW

Company

805-499-0335

www.optodiode.com

OSI Optoelectronics Inc.

+1 310-355-2812

www.osioptoelectr onics.com

Power Technology, Inc.

501.407.0712

www.powertechnology.com
Prizmatix

1 248-436-8085

www.prizmatix.com

retroDIODE LLC

816-721-2517

www.retrodiode.com

Siskiyou Corporation

541-479-8697

www.siskiyou.com

tec5usa

516-653-2000

www.tec5usa.com

Ted Pella, Inc.

800-237-3526

www.tedpella.com

TOFRA, Inc.

650-387-5497

www.tofrainc.net/

UNITRON, Ltd.

631-543-2000

www.unitronusa.com

Vee Gee Scientific

800-423-8842

www.veegee.com

\section{LED/Laser Spinning Disk Imager}

CrESTsrl www.crestopt.com

\section{Lenses}

Abrisa Technologies

+1 (877) 622-7472

www.abrisatechnologies.com

Archer OpTx, Inc.

972-722-1064

www.archeroptx.com

BOCK OPTRONICS INC.

416-674-2804

www.bockoptronics.ca

DayOptics, Inc.

+8659183215681

www.dayoptics.com

Ealing Catalog

800-295-3220

Www.ealingcatalog.com

GOYO OPTICAL, INC.

+81-48-474-2235

www.goyoptical.com

Guernsey Coating Laboratories, Inc. 805-642-1508

www.guernseycoating.com

Hirox-USA, Inc.

201-342-2600

www.hirox-usa.com

JENOPTIK Optical Systems

561-881-7400

www.jenoptik.com

Mitutoyo America Corp. www.mitutoyo.com

Navitar

585-359-4000

www.navitar.com

nPoint, Inc.

608-824-1770

www.npoint.com

Pantec Engineering AG

+423377 1333

www.pantec-medicallaser.com
Perkins Precision Developments, LLC 970-785-2706

www.perkinsprecision.com

Rainbow Research Optics, Inc. (RROI)

303-371-3000

RR-Optics.com

Reynard Corp.

949-366-8866

www.reynardcorp.com

SPOT Imaging Solutions

586-731-6000

www.spotimaging.com

Zygo Corporation

8603478506

www.zygo.com

\section{Light Microscopes (LOMs)}

Abberior Instruments America LLC

+15619729300

www.abberior-instruments-america. com

Absolute Clarity \& Calibration, LLC 860-583-0502

www.absoluteclarity.com

ACCU-SCOPE, Inc.

631-864-1000

www.accu-scope.com

Applied Precision, aGE Healthcare

Company

425-829-9439

www.gelifesciences.com

DiCon Fiberoptics, Inc.

510-620-5167

www.diconfiberoptics.com

Etaluma, Inc.

760-298-2355

www.etaluma.com

Excel Technologies

860-741-3435

www.exceltechnologies.com

FEI

503-726-7500

www.fei.com

Focus e-Beam Technology (Beijing)

Co., Ltd.

86-10-67832505

www.focus-ebeam.com

Hirox-USA, Inc.

201-342-2600

www.hirox-usa.com

Leica Microsystems

800-248-0123

www.leica-microsystems.com

McCrone Microscopes \& Accessories 630-887-7100

www.mccrone.com

Meiji Techno America www.meijitechno.com

MICROS Produktions-und HandelsgmbH www.micros.at

Microscope.com 540-904-0880 www.microscope.com

Microscopy/Microscopy Education (MME) 972-924-5310

www.MicroscopyEducation.com 
Mitutoyo America Corp. www.mitutoyo.com

Nanodyne Measurement Systems, Inc. 651-323-8592

http://www.nano-dyne.com

Nanoscience Instruments, Inc. 888-777-5573

www.nanoscience.com

Siskiyou Corporation 541-479-8697 www.siskiyou.com

TCG, Inc.

925-240-5210

www.tcg-rep.com

Ted Pella, Inc.

800-237-3526

www.tedpella.com

Vee Gee Scientific 800-423-8842

www.veegee.com

Vutara

801-512-2664

www.vutara.com

ZEISS

1-800-233-2343

www.zeiss.com/microscopy mbridge.org/mt

\section{Light Sheet}

Special Optics

973-366-7289

www.specialoptics.com

\section{Light Sheet Microscopy}

Phaseview

+33954030543

www.phaseview.com

Light Tight Imaging Chamber Nor-Cal Products by Pfeiffer Vacuum 800-824-4166

www.n-c.com

Stanford Photonics, Inc.

650-969-5991

www.stanfordphotonics.com

\section{Live-Cell Chambers}

Applied BioPhysics

518-880-6860

www.biophysics.com

Bioptechs

724-282-7145

www.bioptechs.com

Bioscience Tools

1-877-853-9755

www.biosciencetools.com

Charles Supper Company

508-655-4610

www.charles-supper.com

ibidi $\mathrm{GmbH}$

+4989520 46 17-0

www.ibidi.com

Nor-Cal Products by Pfeiffer Vacuum 800-824-4166

www.n-c.com
Live-Cell Imaging

Abberior Instruments America LLC +15619729300

www.abberior-instruments-america. com

ACCU-SCOPE, Inc.

631-864-1000

www.accu-scope.com

Alluxa, Inc.

855-4Alluxa www.alluxa.com

A.P.E Angewandte Physik \& Elektronik $\mathrm{GmbH}$

+4930 986 011-30

www.ape-berlin.de

ASI/Applied Scientific Instrumentation 541-461-8181

www.asiimaging.com

Bioptechs

724-282-7145

www.bioptechs.com

Bruker Nano Surfaces and Metrology 520-741-1044 ext. 1075

www.bruker.com/nano

CANON MEDICAL, Video Sensing

Division

810-357-5022

https://us.medical.canon/VSD

CoolLED

$+44(0) 1264323040$

www.coolled.com

CrestOptics

www.crestopt.com

CytoViva, Inc.

1-334-321-3901

www.cytoviva.com

Etaluma, Inc.

760-298-2355

www.etaluma.com

FEI

503-726-7500

www.fei.com

FEl Munich $\mathrm{GmbH}$ +49898956620

www.fei.com

Nanonics Imaging, Ltd.

866-220-6828

www.nanonics.co.il

Olympus Life Science

(781) 419-3687

www.olympus-lifescience.com

Pembroke Instruments, LLC

415-860-4127

https://pembrokeinstruments.com

Phase Focus Ltd

+441142866377

www.phasefocus.com

Photometrics

520-889-9933

www.photometrics.com

Special Optics

973-366-7289

www.specialoptics.com

Stanford Photonics, Inc.

650-969-5991

www.stanfordphotonics.com
Low Dose Image Acquisition

Advanced Microscopy Techniques 978-774-5550

www.amtimaging.com

\section{Low Temperature Scanning}

Probe Microscopy

NanoMagnetics Instruments Ltd. +441865 522989 www.nanomagnetics-inst.com

\section{Magnetic Field Cancellation}

Field Management Services, Inc. 212-628-6860 x. 205 www.fms-corp.com

Herzan, LLC

949-363-2905

www.herzan.com

Spicer Consulting Limited $+44(0) 1234765773$

www.spicerconsulting.com TMC

800-542-9725

www.techmfg.com

Vibration Engineering Consultants +1 831-465-9189

https://vibeng.com/

\section{Magnification Calibration}

Agar Scientific Ltd +44 (0)1279215 506 www.agarscientific.com

Absolute Clarity \& Calibration, LLC 860-583-0502

www.absoluteclarity.com

APPLIED IMAGE Inc.

585-482-0300

www.appliedimage.com

Geller MicroAnalytical Laboratory, Inc. 978-887-7000 www.gellermicro.com

Ted Pella, Inc. 800-237-3526

www.tedpella.com

\section{Mass Spectrometers/}

Residual Gas Analyzers

Hiden Analytical +44 (0) 1925445225

www.HidenAnalytical.com

MKS Instruments, Inc.

978-645-5500

www.mksinst.com

optek-Danulat, Inc.

262-437-3600

www.optek.com

Pfeiffer Vacuum 603-578-6500 www.pfeiffer-vacuum.com

ULVAC Technologies, Inc.

978-686-7550

www.ulvac.com 
Materials Analysis Services

Materials Analysis Technology Inc. +866-3-611-6678

www.bioma-tek.com

\section{Materials Characterization}

Advanced Surface Microscopy, Inc. 317-895-5630

www.asmicro.com

Anderson Materials Evaluation, Inc. 410-740-8562

www.andersonmaterials.com

Bruker Nano Surfaces and Metrology 520-741-1044 ext. 1075 www.bruker.com/nano

CAMECA

+33143346200

www.cameca.com

Covalent Metrology

408-498-4611

www.covalentmetrology.com

Czitek

888.326.8186

www.czitek.com

DECTRIS Ltd.

+41565002100

www.dectris.com

EDAX, Inc.

201-529-4880

www.edax.com

EAG Laboratories

877-709-9526

www.eag.com

Euclid Technologies, LLC

(301) 637-0669

www.euclidtechlabs.com

Eurofins EAG Laboratories 800-366-3867

www.eag.com

ION-TOF USA, Inc.

845-352-8082

www.iontofusa.com

Janis Research Company, LLC

781-491-0888

www.janis.com

Kammrath \& Weiss

(516) 313-9742

https://www.kammrath-weiss.com/en/

Lake Shore Cryotronics

781-491-0888

www.lakeshore.com/janis

KMLabs, Inc.

303-544-9068

www.kmlabs.com

LeRoy Eyring Center for Solid State

Science

480-727-8578

https://le-csss.asu.edu

Materials Evaluation and Engineering, Inc. 763-449-8870

mee-inc.com

McCrone Associates

630-887-7100

www.mccrone.com

Micron Inc.

302-998-1184

https://microanalytical.com
Park Systems, Inc. 408-986-1110

www.parksystems.com

Psylotech Inc.

+1-847-328-7100

www.psylotech.com

Quantum Design, Inc.

858-481-4400

https://www.qdusa.com/

Renishaw plc.

847-286-9953

www.renishaw.com/raman

TREK, an Advanced Energy Company

1-800-FOR-TREK

www.trekinc.com

Materials Science

ASM International

440-338-5151

asminternational.org

B\&W Tek302-368-7824

www.bwtek.com

Direct Electron, LP

+1 858-384-0291

www.directelectron.com

EAG Laboratories

877-709-9526

www.eag.com

HelioWorks, Inc.

707-578-7200

www.helioworks.com

Kammrath \& Weiss

(516) 313-9742

https://www.kammrath-weiss.com/en/

McCrone Associates

630-887-7100

www.mccrone.com

Meggitt Piezo Technologies

317-876-4670

www.piezotechnologies.com

PIKE Technologies, Inc.

608-271-2721

www.piketech.com

Xradia, Inc.

925-701-3600

www.xradia.com

Measuring Microscopes

Absolute Clarity \& Calibration, LLC 860-583-0502

www.absoluteclarity.com

Capovani Brothers Inc.

518-346-8347

www.capovani.com

Dunwell Tech

888-668-2442

http://dinolite.us/

Excel Technologies860-741-3435 www.exceltechnologies.com

Hirox-USA, Inc.

201-342-2600

www.hirox-usa.com

Motion X Corporation

805-968-2001

www.motionxcorp.com

McCrone Microscopes \& Accessories 630-887-7100

www.mccrone.com
Mitutoyo America Corp. www.mitutoyo.com

Nili Walp

860-355-3776

www.visioneng.us

Semilab USA LLC

+1-508-647-8411

http://www.semilab.com

UNITRON, Ltd.

631-543-2000

www.unitronusa.com

Vision Engineering, Inc.

860-355-3776

www.visioneng.us

Zygo Corporation

8603478506

www.zygo.com

Megapixel Cameras

(See Camera/Digital Camera Systems)

\section{Mercury Lamps}

BLC America

714-889-4116

www.blc-america.com

Bulbworks, Inc.973-584-7171

www.bulbworks.com

UVP LLC

909-946-3197

www.uvp.com

Metallization Service

METRIGRAPHICS, LLC 800-261-2557

http://www.metrigraphicsllc.com/

\section{Metallographic Equipment}

Buehler

www.buehler.com

Excel Technologies

860-741-3435

www.exceltechnologies.com

LECO Corporation

269-985-5496

www.leco.com

Mager Scientific, Inc.

734-426-3885

www.magersci.com

Struers Inc.

440-871-00 71

www.struers.com

Ted Pella, Inc.

800-237-3526

www.tedpella.com

\section{Metallographic Microscopes}

Excel Technologies

860-741-3435

www.exceltechnologies.com

Herzan, LLC

949-363-2905

www.herzan.com

LECO Corporation

269-985-5496

www.leco.com

Mager Scientific, Inc.

734-426-3885

www.magersci.com 
Meiji Techno America www.meijitechno.com

MICROS Produktions-und HandelsgmbH www.micros.at

Microscope.com 540-904-0880 www.microscope.com

Struers Inc. 440-871-00 71 www.struers.com

Ted Pella, Inc. 800-237-3526 www.tedpella.com

UNITRON, Ltd. 631-543-2000 www.unitronusa.com ZEISS

1-800-233-2343

www.zeiss.com/microscopy

\section{Metrology Instruments}

attocube systems

+49-89-2877-809-0

www.attocube.com

Bruker Nano Surfaces and Metrology 520-741-1044 ext.1075 www.bruker.com/nano

Capovani Brothers Inc. 518-346-8347 www.capovani.com

Charles Supper Company 508-655-4610 www.charles-supper.com

Filmetrics, A KLA Company 858-573-9300 www.filmetrics.com

Gamma Scientific 858-279-8034 www.gamma-sci.com

HEIDENHAIN Corporation 847-490-1191 www.heidenhain.us

KEYENCE Corporation of America 888-539-3623 www.keyence.com

Lumencor 503-213-4269 www.lumencor.com

Mitutoyo America Corp. www.mitutoyo.com

MTIInstruments/Fullam www.mtiinstruments.com

Nili Walp 860-355-3776 www.visioneng.us optek-Danulat, Inc. 262-437-3600 www.optek.com

Optikos Corporation 617-354-7557 www.optikos.com
Park Systems, Inc. 408-986-1110 www.parksystems.com

Seron Technologies, Inc. 82-031-349-1411 www.serontech.co.kr

SmarAct Inc. 415-766-9006 www.smaract.com

Vision Engineering, Inc. 860-355-3776 www.visioneng.us

Zygo Corporation 8603478506 www.zygo.com

Micro Balance Citizen Scale Inc. 732-744-1440 www.citizeninc.com

\section{Micro-CT Scanning}

Carestream Molecular Imaging 203-786-5600 www.carestream.com

Deben +44 (0) 1359244 870; 12014105028 www.deben.co.uk

FEI

\section{3-726-7500}

www.fei.com

Micro Photonics 610-366-7103 www.microphotonics.com

Object Research Systems 514-843-3861 www.theobjects.com

Psylotech Inc. +1-847-328-7100 www.psylotech.com

Sigray, Inc. 925-446-4183 http://www.sigray.com

Xradia, Inc. 925-701-3600 www.xradia.com XRE

+32 (0)9 3946794 http://xre.be

\section{Micro Milling Machine}

3D-Micromac AG +49 371 40043-222 www.3d-micromac.com

Clark-MXR, Inc. 734-426-2803 www.cmxr.com

\section{Micro Tools}

MiTeGen 1-607-266-8877 www.mitegen.com

Ted Pella, Inc. 800-237-3526 www.tedpella.com
Micro X-ray Fluorescence

Bruker Corporation 608-276-3000 www.bruker.com

Bruker Nano Analytics 1-800-234-3028 www.bruker.com/nano-analysis

EDAX, Inc. 201-529-4880 www.edax.com

IXRF, Inc. 512-386-6100 www.ixrfsystems.com Sigray, Inc. 925-446-4183 http://www.sigray.com

\section{Micro- and Nanomanipulators} Imina Technologies www.imina.ch

\section{Microanalysis Services}

Advanced MicroBeam, Inc. 330-394-1255 www.advancedmicrobeam.com Anderson Materials Evaluation, Inc. 410-740-8562 www.andersonmaterials.com

CAMCOR

541-346-6447

www.camcor.uoregon.edu

LeRoy Eyring Center for Solid State

Science

480-727-8578

https://le-csss.asu.edu

MAX Detector Repair Group LLC 608-829-0110 maxdetector.com

McCrone Associates 630-887-7100 www.mccrone.com

Micron Inc.

302-998-1184

https://microanalytical.com

Micro Photonics 610-366-7103 www.microphotonics.com

Semion 503-332-9668 www.semionco.com

Wolfram Research, Inc. 217-398-0700 https://www.wolfram.com

\section{Microelectromechanical} Systems (MEMS)

Euclid Technologies, LLC (301) 637-0669 www.euclidtechlabs.com

Quantum Composers Inc 406-582-0227 Quantumcomposers.com

YW MEMS

+86-512-6726-6051

http://www.cleansin.com/ 
Microinjectors

Bunton Instrument Co., Inc. 301-831-3434

www.buntgrp.com

\section{Micromanipulators}

AutoMate Scientific 510-845-6283 www.autom8.com

Kammrath \& Weiss (516) 313-9742 https://www.kammrath-weiss.com/en/

Minitool, Inc. 408-395-1585 www.minitoolinc.com

Nanoscience Instruments, Inc. 888-777-5573 www.nanoscience.com

Prior Scientific, Inc. 781-878-8442 www.prior.com

Siskiyou Corporation 541-479-8697 www.siskiyou.com

Sutter Instrument 415-883-0128 www.sutter.com

\section{Microscope Enclosures}

Field Management Services, Inc. 212-628-6860 x. 205 www.fms-corp.com

HEMCO Corporation 18007794362 www.HEMCOcorp.com

Vibration Engineering Consultants +1 831-465-9189 https://vibeng.com/

\section{Microscope Immersion}

Special Optics 973-366-7289 www.specialoptics.com

\section{Microscope Immersion Oil}

Ted Pella, Inc. 800-237-3526

www.tedpella.com

\section{Microscope Slides}

Chroma Technology Corp. 800-824-7662 www.chroma.com

Globe Scientific Inc. www.globescientific.com

HTA Photomask 408 452-5500 www.htaphotomask.com

Ted Pella, Inc. 800-237-3526 www.tedpella.com UQG (Lab Glasses) + 44 (0) 1223420329 www.uqgoptics.com
Microscope Stages

ASI/Applied Scientific Instrumentation 541-461-8181 www.asiimaging.com

Bunton Instrument Co., Inc. 301-831-3434 www.buntgrp.com

Deben +44 (0) 1359244 870; 12014105028 www.deben.co.uk

Hirox-USA, Inc. 201-342-2600 www.hirox-usa.com

Instec, Inc.

303-444-4608 www.instec.com

Linkam Scientific Instruments +44 (0) 1737363476 www.linkam.co.uk

Mad City Labs, Inc. 608-298-0855 www.madcitylabs.com

nPoint, Inc.

608-824-1770 www.npoint.com

Optimal Engineering Systems, Inc. 888-777-1826 www.oesincorp.com

PIEZOCONCEPT $+33(0) 478742456$ www.piezoconcept.com

PI (Physik Instrumente), LP 508-832-3456 www.pi-usa.us/microscopy

Prior Scientific, Inc. 781-878-8442 www.prior.com

Siskiyou Corporation 541-479-8697 www.siskiyou.com

Sutter Instrument 415-883-0128 www.sutter.com

TOFRA, Inc.

650-387-5497

www.tofrainc.net/

\section{Microscope Stands}

ASI/Applied Scientific Instrumentation 541-461-8181 www.asiimaging.com

Hirox-USA, Inc. 201-342-2600 www.hirox-usa.com

\section{Microscope Viewing Heads}

Nili Walp 860-355-3776

www.visioneng.us

Z\&Z Optoelectronics Tech Co., Ltd. 86-28-85139768 www.zzoptic.com

\section{Microscopy Applications}

Alluxa, Inc. 855-4Alluxa www.alluxa.com
CytoViva, Inc. 1-334-321-3901 www.cytoviva.com

Czitek 888.326.8186 www.czitek.com

Etaluma, Inc. 760-298-2355 www.etaluma.com

Euresys Inc +19497430612 www.euresys.com

Finger Lakes Instrumentation 585-624-3760 www.flicamera.com

GIGAmacro 415-841-3322 www.gigamacro.com

Pembroke Instruments, LLC 415-860-4127 https://pembrokeinstruments.com PCO-TECH Inc.

$$
+1 \text { (248) } 2768820
$$

www.pco-tech.com

Phase Focus Ltd +441142866377 www.phasefocus.com

Spectrolight Inc. 949-800-7780 www.spectrolightinc.com

TechnoSpex Pte Ltd +6562766928 www.technospex.com

\section{Microscopy Education}

Cell Image Library (858) 534-0276 http://www.cellimagelibrary.org/home Hooke College of Applied Sciences 630-887-7100 www.hookecollege.com

Imaging and Analysis, LLC 651-788-2261 www.imagingandanalysis.com

Lehigh Microscopy School 610-758-1112 www.lehigh.edu/microscopy

McCrone Atlas of Microscopic Particles 630-887-7100 www.mccroneatlas.com

McCrone Research Institute 312-842-7100 www.mcri.org

MicroSpec On-site 972-924-5310 microspec-onsite.com

\section{Microscopy Services CAMCOR} 541-346-6447 www.camcor.uoregon.edu Hirox-USA, Inc. 201-342-2600 www.hirox-usa.com 
Martin Microscope Company 864-242-3424

www.MartinMicroscope.com

McCrone Associates 630-887-7100

www.mccrone.com

McCrone Research Institute, Chicago 312-842-7100 www.mcri.org

Meadowlark Optics 303-833-4333 www.meadowlark.com

Norsam Technologies, Inc. 505-984-1133 www.norsam.com

Semion

503-332-9668

www.semionco.com

\section{Microscopy Training}

Lehigh Microscopy School 610-758-1112 www.lehigh.edu/microscopy

McCrone Research Institute 312-842-7100 www.mcri.org

\section{Microtomes and Ultramicrotomes}

Boeckeler Instruments, Inc. 520-745-0001 www.boeckeler.com

Micro Star Technologies 800-533-2509 www.microstartech.com

MICROS Produktions-und HandelsgmbH www.micros.at

Microtome Service Company 315-451-1404 www.microtomeserviceco.com

RMC Boeckeler 1-520-745-0001 www.rmcboeckeler.com

Ted Pella, Inc. 800-237-3526 www.tedpella.com

\section{Microtome Repair}

Microtome Service Company 315-451-1404 www.microtomeserviceco.com

\section{Microwave Heating}

Microwave Research and Applications, Inc.

630-480-7456

www.microwaveresearch.com

\section{Microwave Tissue Processing}

Electron Microscopy Sciences 215-412-8400 www.emsdiasum.com

Energy Beam Sciences, Inc. (860) 653-0411

www.ebsciences.com
Microwave Research and Applications, Inc.

630-480-7456

www.microwaveresearch.com

Ted Pella, Inc.

800-237-3526

www.tedpella.com

\section{Milling}

Verder Scientific Inc (267) 757-0351

www.verder-scientific.com

\section{Moisture Balance}

Citizen Scale Inc.

732-744-1440

www.citizeninc.com

Kett US

www.kett.com

\section{Multiphoton Microscopes}

Alluxa, Inc. 855-4Alluxa www.alluxa.com

A.P.E Angewandte Physik \& Elektronik $\mathrm{GmbH}$

+4930 986 011-30

www.ape-berlin.de

Cycle $\mathrm{GmbH}$

+4940899889120

www.cyclelasers.com

Bruker AFM Probes 520-741-1044

www.brukerafmprobes.com

Bruker Fluorescence Microscopy 608-662-0022

http://www.bruker.com/products/ surface-analysis/fluorescenceoptical- microscopy.html

Bruker Nano Surfaces and Metrology 520-741-1044 ext. 1075 www.bruker.com/nano

KMLabs, Inc.

303-544-9068

www.kmlabs.com

Miltenyi Biotec B.V. \& Co. KG $+49220483060$ www.miltenyibiotec.com

Olympus Life Science 781) 419-3687 www.olympus-lifescience.com

Phaseview +33954030543 www.phaseview.com

Sutter Instrument 415-883-0128 www.sutter.com

VEROPTICS

1-800-939-6627

www.veroptics.com

\section{Multiprobe Imaging}

Access Optics

918-294-1234

www.accessoptics.com
A.P.E Angewandte Physik \& Elektronik $\mathrm{GmbH}$

+4930 986 011-30

www.ape-berlin.de

Nanonics Imaging, Ltd.

1-866-220-6828

www.nanonics.co.il

\section{Nano Indentation}

Bruker Nano Surfaces and Metrology 520-741-1044 ext. 1075 www.bruker.com/nano

Covalent Metrology 408-498-4611 www.covalentmetrology.com

Fischer Technology, Inc. 860-683-0781 www.fischer-technology.com

Hysitron, a Bruker Company 952-835-6366 www.hysitron.com

Micro Star Technologies 800-533-2509 www.microstartech.com

Nanomechanics, Inc., a KLA Tencor

Company 865-978-6490 www.nanomechanicsinc.com

Nanonics Imaging, Ltd. 866-220-6828 www.nanonics.co.il

Nanoscience Instruments, Inc. 888-777-5573 www.nanoscience.com

Norsam Technologies, Inc. 505-984-1133 www.norsam.com

Semilab USA LLC +1-508-647-8411 http://www.semilab.com

\section{Nanomanipulators}

Oxford Instruments NanoAnalysis USA +44 (0) 1494442255 https://nano.oxinst.com/

\section{Nano-Particle Size Analyzer}

NanoAndMore USA, Inc. 843-521-11 08 www.nanoandmore.com

\section{Nanopositioners \& Stages}

Aerotech, Inc. +1-412-963-7470 www.aerotech.com

ASI/Applied Scientific Instrumentation 541-461-81 81 www.asiimaging.com

Charles Supper Company 508-655-4610 www.charles-supper.com 
Imina Technologies www.imina.ch

Kleindiek Nanotechnik $+4971213453950$ www.kleindiek.com

Mad City Labs, Inc. 608-298-0855 www.madcitylabs.com

Nanonics Imaging, Ltd. 866-220-6828 www.nanonics.co.il

Nanoscience Instruments, Inc. 888-777-5573 www.nanoscience.com

nPoint, Inc.

608-824-1770 www.npoint.com

Optimal Engineering Systems, Inc. 888-777-1826

www.oesincorp.com

PIEZOCONCEPT $+33(0) 478742456$ www.piezoconcept.com

PI (Physik Instrumente), LP 508-832-3456 www.pi-usa.us/microscopy

Prior Scientific, Inc. 781-878-8442 www.prior.com

SmarAct Inc.

415-766-9006

www.smaract.com

\section{Nanoprobes/Mechanical} Microprobes

Advent Research Materials Ltd +44 (0) 1865884440 www.advent-rm.com

NenoVision +420605287732

www.nenovision.com

\section{Nanoprobes/Mechanical Microprobes}

Advent Research Materials Ltd +44 (0) 1865884440 www.advent-rm.com

NenoVision $+420605287732$ www.nenovision.com

Carbon Design Innovations 650-697-7070 www.carbondesigninnovations.com

Micro Star Technologies 800-533-2509 www.microstartech.com Nanomechanics, Inc., a KLA Tencor

Company

865-978-6490 www.nanomechanicsinc.com

Nanonics Imaging, Ltd. 866-220-6828 www.nanonics.co.il

Nanoscience Instruments, Inc. 888-777-5573

www.nanoscience.com
Norsam Technologies, Inc. 505-984-1133

www.norsam.com

Sonics \& Materials, Inc. 203-270-4600 www.sonics.com

Xradia, Inc. 925-701-3600 www.xradia.com

\section{Nanoprobes \& Probe Systems}

Angstrom Scientific, Inc. 201-962-7222

www.angstrom.us

\section{Nanosensors}

Access Optics

918-294-1234

www.accessoptics.com

Advent Research Materials Ltd +44 (0) 1865884440 www.advent-rm.com

NanoAndMore USA, Inc. 843-521-1108 www.nanoandmore.com

PI (Physik Instrumente), LP 508-832-3456

www.pi-usa.us/microscopy

\section{Nanotools}

NanoAndMore USA, Inc. 843-521-1108 www.nanoandmore.com

\section{Near-Field Scanning Optical Microscopes (NSOMs)}

Anasys Instruments 805-730-3310 www.anasysinstruments.com

A.P.E. Research SRL $+390402528164$ www.aperesearch.com

Bruker Nano Surfaces and Metrology 520-741-1044 ext. 1075 www.bruker.com/nano

Mad City Labs, Inc. 608-298-0855 www.madcitylabs.com

Nanonics Imaging, Ltd. 866-220-6828 www.nanonics.co.il

New and Used Equipment

ATAGO U.S.A., Inc.

425-637-2107

http://www.atago-usa.com

Hirox-USA, Inc.

201-342-2600

www.hirox-usa.com

J. Kraft Microscopy Services, Inc. 716-592-4402

jkraftmicro.com
SEMTech Solutions, Inc.

978-663-9822

www.semtechsolutions.com

\section{NIR Composition Analyzers}

Bruker Optics

978-663-3660

www.bruker.com

HelioWorks, Inc. 707-578-7200

www.helioworks.com

Kett US

www.kett.com

Malvern Panalytical +1800 279-7297

www.malvernpanalytical.com

\section{NIR Moisture Meter}

HelioWorks, Inc. 707-578-7200 www.helioworks.com

Kett US www.kett.com

\section{Objective Lenses}

Access Optics 918-294-1234 www.accessoptics.com

ACCU-SCOPE, Inc. 631-864-1000 www.accu-scope.com

Beck Optronic Solutions Limited +44 (0) 1442255755 www.beckoptronic.com

JENOPTIK Optical Systems 561-881-7400 www.jenoptik.com

PIKE Technologies, Inc. 608-271-2721 www.piketech.com

Rainbow Research Optics, Inc. (RROI) 303-371-3000

RR-Optics.com

Special Optics 973-366-7289 www.specialoptics.com

UQG (Lab Glasses) + 44 (0) 1223420329 www.uqgoptics.com

Z\&Z Optoelectronics Tech Co., Ltd. 86-28-85139768 www.zzoptic.com

\section{Optical Filters}

Abrisa Technologies +1 (877) 622-7472 www.abrisatechnologies.com

Access Optics 918-294-1234 www.accessoptics.com

Alluxa, Inc. 855-4Alluxa www.alluxa.com 


\section{A Guide to Microscopy Products}

Archer OpTx, Inc. 972-722-1064 www.archeroptx.com

Chroma Technology Corp. 800-824-7662

www.chroma.com

DayOptics, Inc. +8659183215681 www.dayoptics.com

Deposition Sciences, Inc. 707-573-6700

www.depsci.com

DiCon Fiberoptics, Inc. 510-620-5167 www.diconfiberoptics.com

Ealing Catalog 800-295-3220 www.ealingcatalog.com

Evaporated Coatings, Inc. 215-659-3080 www.evaporatedcoatings.com

Guernsey Coating Laboratories, Inc. 805-642-1508 www.guernseycoating.com Iridian Spectral Technologies 613-741-4513 www.iridian.ca

JENOPTIK Optical Systems 561-881-7400 www.jenoptik.com

Laser 2000 (UK) Ltd. +44 (0) 01933461666 www.laser2000.co.uk

Laser Components USA, Inc. (603) 8217040

www.lasercomponents.com

Meadowlark Optics 303-833-4333 www.meadowlark.com

Midwest Optical Systems (MidOpt) (847) 359-3550

www.midopt.com

MLD Technologies, LLC

(650) 938-4485

www.mldtech.com

Moxtek, Inc. 801-225-0930 www.moxtek.com

Optics Balzers 303-502-5401 www.opticsbalzers.com

Omega Optical, LLC 802-251-7300 www.omegafilters.com

Perkins Precision Developments, LLC 970-785-2706 www.perkinsprecision.com

Photon etc. 514-385-9555 www.photonetc.com
Precision Glass \& Optics (PG\&Oß) 714-540-0126

www.pgo.com

Rainbow Research Optics, Inc. (RROI) 303-371-3000

RR-Optics.com

Reynard Corp. 949-366-8866 www.reynardcorp.com

Spectrolight Inc. 949-800-7780 www.spectrolightinc.com

tec5usa 516-653-2000 www.tec5usa.com

UQG (Lab Glasses) + 44 (0) 1223420329 www.uqgoptics.com

Z\&Z Optoelectronics Tech Co., Ltd. 86-28-85139768 www.zzoptic.com

\section{Optical Prototyping System}

Archer OpTx, Inc. 972-722-1064 www.archeroptx.com

Goldstone Scientific LLC +1 248-424-9390 www.GoldstoneScientific.com

McPherson Instruments 978-256-4512 www.McPhersonInc.com

Optikos Corporation 617-354-7557 www.optikos.com

Prizmatix

$1248-436-8085$

www.prizmatix.com

Optical Targets, Reticles, Encoders

METRIGRAPHICS, LLC 800-261-2557

http://www.metrigraphicsllc.com/

\section{Optoelectronic Materials}

Marktech Optoelectronics, Inc. (518) 956-2980 https://www.marktechopto.com

\section{Optogenetics}

Bruker Fluorescence Microscopy 608-662-0022

http://www.bruker.com/products/ surface-analysis/fluorescenceoptical- microscopy.html

\section{Orientation and Phase}

Analysis, TEM

NanoMEGAS 480-389-6816 www.nanomegasusa.com
Particle Analyzer

COXEM CO., LTD

+82-42-861-1686

www.coxem.com

Fluid Imaging Technologies, Inc. 207-846-6100 www.fluidimaging.com

Malvern Panalytical +1 800 279-7297 www.malvernpanalytical.com

\section{Particle Characterization}

\section{Services}

Dune Sciences, Inc. 541-359-1959 www.dunesciences.com

Eurofins EAG Laboratories 800-366-3867 www.eag.com

McCrone Associates 630-887-7100 www.mccrone.com

McCrone Atlas of Microscopic Particles 630-887-7100 www.mccroneatlas.com

Particle Technology Labs 630-969-2703 www.particletechlabs.com

Verder Scientific Inc (267) 757-0351 www.verder-scientific.com

\section{Perfusion System}

AutoMate Scientific 510-845-6283 www.autom8.com

Bioptechs 724-282-7145 www.bioptechs.com

Bioscience Tools 1-877-853-9755 www.biosciencetools.com

\section{Phase Contrast Microscopy}

ACCU-SCOPE, Inc.

631-864-1000

www.accu-scope.com

\section{Phase Identification}

International Centre for Diffraction Data (ICDD)

610-325-9814

www.icdd.com

NanoMEGAS

480-389-6816

www.nanomegasusa.com

neaspec $\mathrm{GmbH}$ 49-176-2320-3996

www.neaspec.com

Xradia, Inc.

925-701-3600

www.xradia.com

\section{Photography Supplies}

Carestream Molecular Imaging 203-786-5600

www.carestream.com 


\section{A Guide to Microscopy Products}

Ted Pella, Inc.

800-237-3526

www.tedpella.com

\section{Photometers}

optek-Danulat, Inc.

262-437-3600

www.optek.com

\section{Piezo Stages and Controllers}

Aerotech, Inc.

+1-412-963-7470

www.aerotech.com

attocube systems

$+49-89-2877-809-0$

www.attocube.com

Mad City Labs, Inc.

608-298-0855

www.madcitylabs.com

nPoint, Inc.

608-824-1770

www.npoint.com

Prior Scientific, Inc.

781-878-8442

www.prior.com

SmarAct Inc.

415-766-9006

www.smaract.com

TREK, an Advanced Energy Company 1-800-FOR-TREK

www.trekinc.com

Voxa

415-858-0393

http://projectvoxa.com

\section{Plasma Cleaners}

Anatech USA

775-657-8685

www.anatechusa.com

CryoSol-World BV

$+31(0) 433022472$

www.cryosol-world.com

E. A. Fischione Instruments, Inc.

724-325-5444

www.fischione.com

Evactron $\AA$ by XEI Scientific, Inc.

650-369-0133

www.evactron.com

Glow Research

480-621-8405

www.glowresearch.org

ibss Group, Inc.

650-513-1488

www.ibssgroup.com

Quorum Technologies, Ltd.

$+44(0) 1323810981$

www.quorumtech.com

SPI Supplies

610-436-5400

www.2spi.com

Ted Pella, Inc.

800-237-3526

www.tedpella.com
Pocket Microscope

ioLight Magnificent Mobile

Microscopes

+44 (0)333202 7101

ioLight.co.uk

Polarized Light Microscopy

LASERGLOW Technologies

+1416-729-7976

www.laserglow.com

LW Scientific

800-726-7345

www.Iwscientific.com

McCrone Research Institute

312-842-7100

www.mcri.org

McCrone Research Institute, Chicago

312-842-7100

www.mcri.org

Meadowlark Optics

303-833-4333

www.meadowlark.com

Meiji Techno America

Microscopy/Microscopy Education (MME)

972-924-5310

www.MicroscopyEducation.com

UNITRON, Ltd.

631-543-2000

www.unitronusa.com

\section{Polarizers}

Abrisa Technologies

+1 (877) 622-7472

www.abrisatechnologies.com

Chroma Technology Corp.

800-824-7662

www.chroma.com

DayOptics, Inc.

+8659183215681

www.dayoptics.com

Laser Components USA, Inc.

(603) 8217040

www.lasercomponents.com

MLD Technologies, LLC

(650) 938-4485

www.mldtech.com

Moxtek, Inc.

801-225-0930

www.moxtek.com

Precision Glass \& Optics (PG\&O $®)$

714-540-0126

www.pgo.com

Rainbow Research Optics, Inc. (RROI) 303-371-3000

RR-Optics.com

Reynard Corp.

949-366-8866

www.reynardcorp.com

\section{Polishing Consumables}

Buehler

www.buehler.com

Dianamic Abrasive Products Inc. 248-280-1185

awww.dianamic.com
Excel Technologies

860-741-3435

www.exceltechnologies.com

Ted Pella, Inc.

800-237-3526

www.tedpella.com

\section{Polishing Equipment}

Excel Technologies 860-741-3435

www.exceltechnologies.com

LECO Corporation

269-985-5496

www.leco.com

Mager Scientific, Inc.

734-426-3885

www.magersci.com

Struers Inc.

440-871-0071

www.struers.com

Ted Pella, Inc.

800-237-3526

www.tedpella.com

Portable Microscope

ioLight Magnificent Mobile

Microscopes

+44 (0)3332027101

ioLight.co.uk

\section{Positioning Systems}

Aerotech, Inc.

$$
+1-412-963-7470
$$

www.aerotech.com

Finger Lakes Instrumentation 585-624-3760

www.flicamera.com

Haydon Kerk Motion Solutions, Inc. 203-756-7441

www.haydonkerkpittman.com

Motion X Corporation

805-968-2001

www.motionxcorp.com

Optimal Engineering Systems, Inc.

888-777-1826

www.oesincorp.com

SmarAct Inc.

415-766-9006

www.smaract.com

TEMWindows

585-214-0585

www.TEMWindows.com

Velmex, Inc.

585-657-6151

www.velmex.com

\section{Powder Blenders}

Glen Mills Inc.

973-777-0777

www.GLENMILLS.com

Precision Positioning Stages

Mad City Labs, Inc.

608-298-0855

www.madcitylabs.com 
Prepared Slides

Chroma Technology Corp. 800-824-7662

www.chroma.com

\section{Prisms}

\section{(see Beam Splitters)}

Proportional Counter Windows

Moxtek, Inc.

801-225-0930

www.moxtek.com

\section{Publishers}

Cambridge University Press 212-337-5000

www.cambridge.org

Cell Image Library (858) 534-0276

http://www.cellimagelibrary.org/home John Wiley \& Sons, Inc.

781-388-8544

www.wiley.com

Photonics Media/Laurin Publishing 413-499-0514

www.photonics.com

Royal Microscopical Society 01865265760

www.rms.org.uk

Springer Science+Business Media 800-777-4643

www.springer.com

\section{Quartz Slides}

Ted Pella, Inc. 800-237-3526

www.tedpella.com

UQG (Lab Glasses) + 44 (0) 1223420329

www.uqgoptics.com

\section{R\&D Pilot Production Systems}

Archer OpTx, Inc. 972-722-1064 www.archeroptx.com

Clark-MXR, Inc. 734-426-2803 www.cmxr.com

Optikos Corporation 617-354-7557 www.optikos.com

\section{Raman Optical Filters}

Alluxa, Inc. 855-4Alluxa www.alluxa.com

Iridian Spectral Technologies Ltd. 613-741-4513 www.iridian.ca

Perkins Precision Developments, LLC 970-785-2706 www.perkinsprecision.com

\section{Raman Spectroscopy/Microscopy} AIST-NT

www.aist-nt.com

Anasys Instruments 805-730-3310

www.anasysinstruments.com

A.P.E Angewandte Physik \& Elektronik $\mathrm{GmbH}$

+4930 986 011-30

www.ape-berlin.de

Armadillo Sia

(408) 834-7422

https://armadillosia.com

BioTools

561-625-0133

www.btools.com

Bruker Nano Surfaces and Metrology 520-741-1044 ext.

1075

www.bruker.com/nano

Bruker Optics

978-663-3660

www.bruker.com

B\&W Tek

302-368-7824

www.bwtek.com

Chem

Image Corporation

www.chemimage.com

CRAIC Technologies, Inc.

310-573-8180

www.microspectra.com

CRYO Industries of America, Inc.

603-621-9957

www.cryoindustries.com

CytoViva, Inc.

1-334-321-3901

www.cytoviva.com

DiCon Fiberoptics, Inc.

510-620-5167

www.diconfiberoptics.com

Harrick Scientific Products, Inc. www.harricksci.com

HORIBA Scientific

732-494-8660

www.horiba.com/scientific Instec, Inc. 303-444-4608

www.instec.com

Iridian Spectral Technologies Ltd.

613-741-4513

www.iridian.ca

Janis Research Company, LLC

781-491-0888

www.janis.com

Kimmon Koha USA, Inc.

(303) 754-0401

www.kimmon.com

KMLabs, Inc.

303-544-9068

www.kmlabs.com

Lake Shore Cryotronics

781-491-0888

www.lakeshore.com/janis
LeRoy Eyring Center for Solid State Science

480-727-8578

https://le-csss.asu.edu

Linkam Scientific Instruments +44 (0) 1737363476

www.linkam.co.uk

McPherson Instruments

978-256-4512

www.McPhersonlnc.com

Microscopy/Microscopy Education (MME) 972-924-5310

www.MicroscopyEducation.com

MicroSpec On-site

972-924-5310

microspec-onsite.com

Nanonics Imaging, Ltd.

866-220-6828

www.nanonics.co.il

Photon etc.

514-385-95 55

www.photonetc.com

Princeton Instruments

www.princetoninstruments.com

Raptor Photonics

+442828270141

www.raptorphotonics.com

Renishaw plc.

847-286-9953

www.renishaw.com/raman

Rigaku Corporation

+1-281-362-2300

www.rigaku.com

RPMC Lasers, Inc.

(636) 272-7227

https://www.rpmclasers.com

tec5usa

516-653-2000

www.tec5usa.com

TechnoSpex Pte Ltd

+656276 6928

www.technospex.com

UQG (Lab Glasses)

$+44(0) 1223420329$

www.uqgoptics.com

US Fiberoptec Technology Inc.

1 (408) 834-7422

usfiberoptec.com

WITec GmbH

$+49(0) 731140700$

www.witec.de

Recirculating Water Chillers

Applied Thermal Control Ltd.

+44 (0)1530 389998

www.app-therm.com

BV Thermal Systems

20-522-3701

www.BVThermal.com 


\section{Reference Materials/Reference Standards (See Calibration and Reference Standards)}

\section{Ted Pella, Inc. \\ 800-237-3526 \\ www.tedpella.com \\ UNITRON, Ltd. \\ 631-543-2000 \\ www.unitronusa.com}

Reflection Light Microscope

\section{Refractive Index Liquids}

\section{Resharpening Services}

\section{(See Knife Resharpening)}

\section{Resolution Standards}

Agar Scientific Ltd +44 (0)1279 215506

Www.agarscientific.com

APPLIED IMAGE Inc.

585-482-0300

www.appliedimage.com

ARGOLIGHT

+33 (0)564 310850

www.argolight.com

Graticules Optics Ltd +441732360460

www.graticulesoptics.com

HTA Photomask

408 452-5500

www.htaphotomask.com

Ted Pella, Inc.

800-237-3526

www.tedpella.com

\section{Sample Preparation \& Handling}

3D-Micromac AG

+49371 40043-222

www.3d-micromac.com

Buehler

www.buehler.com

CryoSol-World BV

$+31(0) 433022472$

www.cryosol-world.com

DiATOME U.S.

2154128390

www.emsdiasum.com

DELMIC BV

+31 (0)157440158

www.delmic.com

E. A. Fischione Instruments, Inc. 724-325-5444

www.fischione.com

Electron Microscopy Sciences 215-412-8400

www.emsdiasum.com

Evactron $₫$ by XEI Scientific, Inc. 650-369-0133

www.evactron.com

Excel Technologies 860-741-3435 www.exceltechnologies.com

Glen Mills Inc.

973-777-0777

www.GLENMILLS.com ibss Group, Inc.

650-513-1488

www.ibssgroup.com

LatticeGear LLC

+1 (503) 828-0400

www.latticegear.com

McCrone Associates

630-887-7100

www.mccrone.com

Miltenyi Biotec B.V. \& Co. KG

+49220483060

www.miltenyibiotec.com

Minitool, Inc.

408-395-1585

www.minitoolinc.com

PIKE Technologies, Inc.

608-271-2721

www.piketech.com

PRO Scientific

www.proscientific.com

RedWave Technology

203-628-4833

www.redwavetech.com

Sonics \& Materials, Inc.

203-270-4600

www.sonics.com

S.T.Japan-Europe $\mathrm{GmbH}$

+49 (0) 2234-956372

www.stjapan.de

Ted Pella, Inc.

800-237-3526

www.tedpella.com

\section{Sample Storage}

Microscopy Innovation, LLC 888-302-3925

www.microscopyinnovations.com

MiTeGen

1-607-266-8877

www.mitegen.com

Ted Pella, Inc.

800-237-3526

www.tedpella.com

\section{Scanners (SPM)}

NanoMagnetics Instruments Ltd. +441865 522989 www.nanomagnetics-inst.com

Nanonics Imaging, Ltd.

866-220-6828

www.nanonics.co.il

nPoint, Inc.

608-824-1770

www.npoint.com

\section{Scanning Electron}

Microscopes (SEMs)

Anatech USA

775-657-8685

www.anatechusa.com

Angstrom Scientific, Inc.

201-962-7222

www.angstrom.us

DELMIC BV

+31 (0)157440158

www.delmic.com
Delong America

1-866-335-6648

www.lv-em.com

COXEM CO., LTD

+82-42-861-1686

www.coxem.com

Electron Optics Instruments LLC 973-220-8689

www.eoillc.com

Euclid Technologies, LLC

(301) 637-0669

www.euclidtechlabs.com

FEI

503-726-7500

www.fei.com

Focus e-Beam Technology (Beijing)

Co.,Ltd.

86-10-67832505

www.focus-ebeam.com

Hitachi High Technologies America, Inc. 800-253-3053

www.hitachi-hightech.com/us

JEOL USA

978-535-5900

www.jeolusa.com

J. Kraft Microscopy Services, Inc.

716-592-4402

jkraftmicro.com

Lehigh Microscopy School

610-758-1112

www.lehigh.edu/microscopy

McCrone Microscopes \& Accessories

630-887-7100

www.mccrone.com

Nanolmages, LLC

866-601-6266

www.nanoimages.com

Nanoscience Instruments, Inc.

888-777-5573

www.nanoscience.com

Nion Co.

425-822-1521

www.nion.com

Pemtron Corporation

82-2-2627-3780

www.pemtron.com

SAES Getters USA, Inc.

719-576-3200

www.saes-group.com

SEMTech Solutions, Inc.

978-663-9822

www.semtechsolutions.com

Seron Technologies Inc.

82-031-349-1411

www.serontech.co.kr

Tescan

724-772-7433

www.tescan.com

TSS Microscopy

+15036164710

http://www.tssmicroscopy.com/

Voxa

415-858-0393

http://projectvoxa.com

ZEISS

1-800-233-2343

www.zeiss.com/microscopy 


\author{
Scanning Electron \\ Microscopy (SEM) \\ Lehigh Microscopy School \\ 610-758-1112 \\ www.lehigh.edu/microscopy \\ Raith America, Inc. \\ 631-738-9500 \\ www.raith.com
}

\section{Scanning Electron \\ Microscopy (SEM) Courses}

Hooke College of Applied Sciences 630-887-7100

www.hookecollege.com

Lehigh Microscopy School

610-758-1112

www.lehigh.edu/microscopy

McCrone Research Institute

312-842-7100

www.mcri.org

McCrone Research Institute, Chicago

312-842-7100

www.mcri.org

\section{Scanning Probe}

Microscopes (SPMs)

AFMWorkshop

888-671-5539

www.afmworkshop.com

Anasys Instruments

805-730-3310

www.anasysinstruments.com

A.P.E. Research SRL

$+390402528164$

www.aperesearch.com

Asylum Research, an Oxford Instruments Company

805-696-6466

www.oxford-instruments.com/AFM

attocube systems

+49-89-2877-809-0

www.attocube.com

Bruker Nano Surfaces and Metrology 520-741-1044 ext. 1075

www.bruker.com/nano

ION-TOF USA, Inc.

845-352-8082

www.iontofusa.com

Nanonics Imaging, Ltd.

866-220-6828

www.nanonics.co.il

Nanosurf AG

+41619274747

www.nanosurf.com

NenoVision

+420605287732

www.nenovision.com

nPoint, Inc.

608-824-1770

www.npoint.com

Park Systems, Inc.

408-986-1110

www.parksystems.com

Quantum Design, Inc.

858-481-4400

https://www.qdusa.com/afsem.html
Semilab USA LLC

+1-508-647-8411

http://www.semilab.com

SPECS

+49-30-4678240

www.specs.com

\section{Scanning Transmission Electron Microscopes (STEMs) \\ Delong America \\ 1-866-335-6648 \\ www.lv-em.com}

Scanning Tunneling Microscopes

A.P.E. Research SRL

$+390402528164$

www.aperesearch.com

Asylum Research, an Oxford Instruments Company

805-696-6466

www.oxford-instruments.com/AFM

SPECS

+49-30-4678240

www.specs.com

\section{Scintillators}

Amptek Inc.

781-275-2242

www.amptek.com

Berkeley Nucleonics Corp

1-415-453-9955

www.berkeleynucleonics.com

M.E. Taylor Engineering, Inc.

301-975-9798

www.semsupplies.com

Ted Pella, Inc.

800-237-3526

www.tedpella.com

\section{Secondary Ion Mass \\ Spectrometer (SIMS) \\ CAMECA}

+33143346200

www.cameca.com

Hiden Analytical

+44 (0) 1925445225

www. HidenAnalytical.com

ION-TOF USA, Inc.

845-352-8082

www.iontofusa.com

Physical Electronics

952-828-6100

www.phi.com

\section{SEM Accessories}

Agar Scientific Ltd

+44 (0)1279 215506

www.agarscientific.com

Anatech USA

775-657-8685

www.anatechusa.com

Applied Physics Technologies, Inc.

503-434-5550

www.a-p-tech.com
COXEM CO., LTD

+82-42-861-1686

www.coxem.com

E. A. Fischione Instruments, Inc. 724-325-5444

www.fischione.com

Electron Optics Instruments LLC 973-220-8689

www.eoillc.com

element $\mathrm{Pi}$

833-314-1593

www.elementpi.com

Ellcie Industries $\mathrm{GmbH}$

+493881755400

www.ellcie.com

Focus e-Beam Technology (Beijing)

Co.,Ltd.

86-10-67832505

www.focus-ebeam.com

Gatan

925-463-0200

www.gatan.com

Hysitron, a Bruker Company

952-835-6366

www.hysitron.com

ibss Group, Inc.

650-513-1488

www.ibssgroup.com

Imina Technologies

www.imina.ch

Integrated Dynamics Engineering IDE

781-326-5700

www.ideworld.com

JC Nabity Lithography Systems

406-587-0848

www.jcnabity.com

J. Kraft Microscopy Services, Inc.

716-592-4402

jkraftmicro.com

Kammrath \& Weiss

(516) 313-9742

https://www.kammrath-weiss.com/en/

Kleindiek Nanotechnik

+4971213453950

www.kleindiek.com

McCrone Microscopes \& Accessories 630-887-7100

www.mccrone.com

M.E. Taylor Engineering, Inc.

301-975-9798

www.semsupplies.com

Minus K Technology, Inc.

310-348-9656

www.minusk.com

MTIInstruments/Fullam

www.mtiinstruments.com

Nanomechanics, Inc., a KLA Tencor

Company

865-978-6490

www.nanomechanicsinc.com

Nanonics Imaging, Ltd.

866-220-6828

www.nanonics.co.il

NenoVision

+420605287732

www.nenovision.com 


\section{A Guide to Microscopy Products}

Pemtron Corporation 82-2-2627-3780

www.pemtron.com

PNDetector $\mathrm{GmbH}$

+4989 309087-100

www.pndetector.de

PNSensor $\mathrm{GmbH}$

(49) 8930908713

www.pnsensor.de

Psylotech Inc.

+1-847-328-7100

www.psylotech.com

Raith America, Inc.

631-738-9500

www.raith.com

Semilab USA LLC

+1-508-647-8411

http://www.semilab.com

SEMTech Solutions, Inc.

978-663-9822

www.semtechsolutions.com

Seron Technologies, Inc.

82-031-349-1411

www.serontech.co.kr

SII Nanotechnology USA, Inc.

www.siintusa.com

Small World, LLC

703-849-1492

www.small-world.net

SPI Supplies

610-436-5400

www.2spi.com

Spicer Consulting Limited

$+44(0) 1234765773$

www.spicerconsulting.com

TMC

800-542-9725

www.techmfg.com

Ted Pella, Inc.

800-237-3526

www.tedpella.com

\section{SEM Analysis Services}

Advanced MicroBeam, Inc.

330-394-1255

www.advancedmicrobeam.com

Anderson Materials Evaluation, Inc. 410-740-8562

www.andersonmaterials.com

Covalent Metrology

408-498-4611

www.covalentmetrology.com

Electron Optics Instruments LLC 973-220-8689

www.eoillc.com

Focus e-Beam Technology (Beijing)

Co.,Ltd.

86-10-67832505

www.focus-ebeam.com

Geller MicroAnalytical Laboratory, Inc.

978-887-7000

www.gellermicro.com

Materials Evaluation and Engineering, Inc.

763-449-8870

mee-inc.com

McCrone Associates

630-887-7100

www.mccrone.com
Nanolmages, LLC

866-601-6266

www.nanoimages.com

Particle Technology Labs

630-969-2703

www.particletechlabs.com

SEMTech Solutions, Inc.

978-663-9822

www.semtechsolutions.com

TSS Microscopy

+1503616 4710

http://www.tssmicroscopy.com/

\section{SEM/STEM Digital}

Imaging Systems

AJU Technology

82-53-352-2582

DECTRIS Ltd.

+41565002100

www.dectris.com

Direct Electron, LP

+1 858-384-0291

www.directelectron.com

EAG Laboratories

877-709-9526

www.eag.com

Gatan

925-463-0200

www.gatan.com

J. Kraft Microscopy Services, Inc.

716-592-4402

jkraftmicro.com

M.E. Taylor Engineering, Inc.

301-975-9798

www.semsupplies.com

PNDetector $\mathrm{GmbH}$

+4989 309087-100

www.pndetector.de

Quartz Imaging Corporation

604-488-3911

www.quartzimaging.com

ResAlta Research Technologies Corp. 303-748-4346

www.resaltatech.com

X-Spectrum GmbH

+4915731711472

https://x-spectrum.de/

\section{SEM and TEM Sample Holders}

Gatan

925-463-0200

www.gatan.com

Hummingbird Scientific

360-252-2737

www.hummingbirdscientific.com

NORCADA INC.

780-431-9637

www.norcada.com

Materials Analysis Technology Inc.

+866-3-611-6678

www.bioma-tek.com

Protochips

919-377-0812

www.protochips.com

Ted Pella, Inc.

800-237-3526

www.tedpella.com
Voxa

415-858-0393

http://projectvoxa.com

Semiconductor Failure Analysis

3D-Micromac AG

+49371 40043-222

www.3d-micromac.com

Covalent Metrology

408-498-4611

www.covalentmetrology.com

Cycle $\mathrm{GmbH}$

+49408998 89120

www.cyclelasers.com

Gatan

925-463-0200

www.gatan.com

Hamamatsu Corporation

908-231-0960

www.hamamatsu.com

HORIBA Scientific

732-494-8660

www.horiba.com/scientific

Materials Evaluation and Engineering, Inc. 763-449-8870

mee-inc.com

Park Systems, Inc.

408-986-1110

www.parksystems.com

Princeton Infrared Technologies, Inc.

609-917-3380

www.princetonirtech.com

SENSORS UNLIMITED INC., A PART OF COLLINS AEROSPACE

609-333-8200

www.sensorsinc.com

Xradia, Inc.

925-701-3600

www.xradia.com

\section{Semi Micro Balance}

Citizen Scale Inc.

732-744-1440

www.citizeninc.com

\section{Service and Repair}

Applied Thermal Control Ltd.

+441530389998

www.app-therm.com

Associated Environmental Systems

978.772.0022

www.AssociatedEnvironmentalSystems. com

Electron Optics Instruments LLC

973-220-8689

www.eoillc.com

FEI

503-726-7500

www.fei.com

Hirox-USA, Inc.

201-342-2600

www.hirox-usa.com

J. Kraft Microscopy Services, Inc.

716-592-4402

jkraftmicro.com 


\section{A Guide to Microscopy Products}

Microtome Service Company 315-451-1404

www.microtomeserviceco.com

SEMTech Solutions, Inc. 978-663-9822

www.semtechsolutions.com

TGS Technologies, LLC

724-453-3865

www.tgstechnologies.net

TSS Microscopy

$+15036164710$

http://www.tssmicroscopy.com/

\section{Service Laboratories}

Advanced MicroBeam, Inc. 330-394-1255

www.advancedmicrobeam.com

Agilent Crosslab, iLab Operations

Software

1-617-297-2805/1-800-690-2957

www.agilent.com/chem/ilab

Anderson Materials Evaluation, Inc. 410-740-8562

www.andersonmaterials.com

CAMCOR

541-346-6447

www.camcor.uoregon.edu

Geller MicroAnalytical Laboratory, Inc. 978-887-7000

www.gellermicro.com

McCrone Associates

630-887-7100

www.mccrone.com

Nanomechanics, Inc., a KLA Tencor

Company

865-978-6490

www.nanomechanicsinc.com

Norsam Technologies, Inc.

505-984-1133

www.norsam.com

Polylnsight LLC www.polyinsight.com

Semion

503-332-9668

www.semionco.com

TSS Microscopy

+15036164710

http://www.tssmicroscopy.com/

\section{Short Courses}

Hooke College of Applied Sciences 630-887-7100

www.hookecollege.com

McCrone Research Institute

312-842-7100

www.mcri.org

Royal Microscopical Society

01865265760

www.rms.org.uk

\section{Silicon Drift Detectors} (SDD) for SEM and TEM

Amptek Inc.

781-275-2242

www.amptek.com
Bruker Nano Analytics

1-800-234-3028

www.bruker.com/nano-analysis

EDAX, Inc.

201-529-4880

www.edax.com

Oxford Instruments

978-369-9933

www.oxinst.com

Oxford Instruments NanoAnalysis USA

+44 (0) 1494442255

https://nano.oxinst.com

PNDetector $\mathrm{GmbH}$

+4989 309087-100

www.pndetector.de

RaySpec Ltd

+44 1628 5330-60

www.rayspec.co.uk

SII Nanotechnology USA, Inc. www.siintusa.com

Thermo Fisher Scientific

(508) 479-6623

thermofisher.com $/ \mathrm{msr}$

\section{Slides and Coverslips}

Bioscience Tools

1-877-853-9755

www.biosciencetools.com

Globe Scientific Inc.

www.globescientific.com

ibidi $\mathrm{GmbH}$

+4989520 46 17-0

www.ibidi.com

Microscope.com

540-904-0880

www.microscope.com

Ted Pella, Inc.

800-237-3526

www.tedpella.com

UQG (Lab Glasses)

+44 (0) 1223420329

www.uqgoptics.com

\section{Small Tools}

Minitool, Inc.

408-395-15 85

www.minitoolinc.com

Norsam Technologies, Inc.

505-984-1133

www.norsam.com

Ted Pella, Inc.

5303395049

www.tedpella.com

TGS Technologies, LLC

724-453-3865

www.tgstechnologies.net

Society and Event Organizer

ASM International

440-338-5151

asminternational.org

MSA MegaBooth

800-538-3672

www.microscopy.org
Software

Pixelink

1-833-247-1211

www.pixelink.com

\section{Software for Microanalysis}

CAMECA

+33143346200

www.cameca.com

Digital Surf

0033381504800

www.digitalsurf.com

Exprodo Software

562-275-1786

www.exprodo.com/calpendo

HREM Research, Inc.

81-493-35-3919

www.hremresearch.com

Image Metrology A/S

+4546923400

www.imagemet.com

Media Cybernetics

301-495-3305

www.mediacy.com

Protochips

919-377-0812

www.protochips.com

Quartz Imaging Corporation 604-488-3911

www.quartzimaging.com

Seron Technologies, Inc.

82-031-349-1411

www.serontech.co.kr

Wolfram Research, Inc.

217-398-0700

https://www.wolfram.com

\section{Software for Microscopy}

Applied Electro-Optics, Inc.

512-686-0752

www.aeousa.com

Black Mesa Imaging, LLC

484-693-0563

www.blackmesa-imaging.com

Chemlmage Corporation www.chemimage.com

Datacolor Inc.

800-982-6499

www.datacolor.com

DigiSENS

www.digisens3d.com

Digital Surf

0033381504800

www.digitalsurf.com

Exprodo Software

562-275-1786

www.exprodo.com/calpendo

FEI

503-726-7500

www.fei.com

FOM Networks, Inc.

224-225-9168

www.fomnetworks.com

Hirox-USA, Inc.

201-342-2600

www.hirox-usa.com 


\section{A Guide to Microscopy Products}

HREM Research, Inc. 81-493-35-3919 www.hremresearch.com Image Metrology A/S +45 46923400 www.imagemet.com

Integrated Dynamics Engineering IDE 781-326-5700

www.ideworld.com

Lumenera Corporation 613-736-4077

www.lumenera.com

Media Cybernetics 301-495-3305 www.mediacy.com

Nion Co.

425-822-1521

www.nion.com

Object Research Systems

514-843-3861

www.theobjects.com

Quartz Imaging Corporation

604-488-3911

www.quartzimaging.com

ResAlta Research Technologies Corp. 303-748-4346

www.resaltatech.com

Small World, LLC

703-849-1492

www.small-world.net

Scientific Volume Imaging

+31356421626

www.svi.n

SPOT Imaging Solutions

586-731-6000

www.spotimaging.com

TVIPS GmbH

49-89-8506567

www.tvips.com

Visage Imaging

858-356-5517

www.visageimaging.com

Vutara

801-512-2664

www.vutara.com

Zarbeco, LLC

973-933-2043

www.zarbeco.com

\section{Spatial Light Modulator}

Holo/Or Ltd.

+972-8-9409687

www.holoor.co.il/

\section{Specimen Holders}

Charles Supper Company

508-655-4610

www.charies-supper.com

E. A. Fischione Instruments, Inc.

724-325-5444

www.fischione.com

Gatan

925-463-0200

www.gatan.com

Hummingbird Scientific

\section{0-252-2737}

www.hummingbirdscientific.com

Materials Analysis Technology Inc.

+866-3-611-6678

www.bioma-tek.com

S.T.Japan-Europe $\mathrm{GmbH}$

+49 (0) 2234-956372

www.stjapan.de

Ted Pella, Inc.

800-237-3526

www.tedpella.com

TGS Technologies, LLC

724-453-3865

www.tgstechnologies.net

Tousimis

301-881-2450

www.tousimis.com

Voxa

415-858-0393

http://projectvoxa.com

\section{Specimen Preparation Equipment}

3D-Micromac AG

+49371 40043-222

www.3d-micromac.com

Buehler

www.buehler.com

CryoSol-World BV

+31(0)43 3022472

www.cryosol-world.com

E. A. Fischione Instruments, Inc.

724-325-5444

www.fischione.com

element $\mathrm{P}$

833-314-1593

www.elementpi.come

Excel Technologies

860-741-3435

www.exceltechnologies.com

EXpressLO LLC

+1-321-663-3806

www.EXpressLO.com

Gatan

925-463-0200

www.gatan.com

LatticeGear LLC

+1 (503) 828-0400

www.latticegear.com

Microscopy Innovations

715-384-3292 www.

microscopyinnovations.com

PRO Scientific

www.proscientific.com

RMC Boeckeler

1-520-745-0001

www.rmcboeckeler.com

S.T.Japan-Europe $\mathrm{GmbH}$

+49 (0) 2234-956372

www.stjapan.de

Ted Pella, Inc.

800-237-35 26

www.tedpella.com
Specimen Preparation

\& Handling

CryoSol-World BV

$+31(0) 433022472$

www.cryosol-world.com

Delaware Diamond Knives, Inc. 800-222-5143

www.ddk.com

E. A. Fischione Instruments, Inc. 724-325-5444 www.fischione.com

Excel Technologies 860-741-3435

www.exceltechnologies.com

EXpressLO LLC

+1-321-663-3806

www.EXpressLO.com

Gatan

925-463-0200

www.gatan.com

Microscopy Innovations

715-384-3292

www.microscopyinnovations.com

Technotrade International, Inc.

603-622-5011

www.technotradeinc.com

Ted Pella, Inc.

800-237-3526

www.tedpella.com

Specimen Preparation Supplies

Delaware Diamond Knives, Inc.

800-222-5143

www.ddk.com

Fischone Instruments

724-325-5444

www.fischone.com

Globe Scientific Inc.

www.globescientific.com

LatticeGear LLC

+1 (503) 828-0400

www.latticegear.com

Technotrade International, Inc.

603-622-5011

www.technotradeinc.com

Ted Pella, Inc.

800-237-3526

www.tedpella.com

Tousimis

301-881-2450

www.tousimis.com

\section{Specimen Stages}

nPoint, Inc.

608-824-1770

www.npoint.com

Kammrath \& Weiss

(516) 313-9742

https://www.kammrath-weiss.com/en/

ResAlta Research Technologies Corp.

303-748-4346

www.resaltatech.com

Voxa

415-858-0393

http://projectvoxa.com 
Spectrometers (Light)

Amptek Inc.

781-275-2242

www.amptek.com

Andor Technology

+442890237126

www.andor.oxinst.com

Applied Spectra, Inc.

510-657-7679

www.appliedspectra.com

Armadillo Sia

(408) 834-7422

https://armadillosia.com

Bruker Optics

978-663-3660

www.bruker.com

CeramOptec Industries, Inc. 413-525-0600

www.ceramoptec.com

CRAIC Technologies, Inc. 310-573-8180

www.microspectra.com

Filmetrics, A KLA Company 858-573-9300

www.filmetrics.com

Hamamatsu Corporation

908-231-0960

www.hamamatsu.com

HORIBA Scientific

732-494-8660

www.horiba.com/scientific

McPherson Instruments

978-256-4512

www.McPhersonlnc.com

neaspec $\mathrm{GmbH}$

49-176-2320-3996

www.neaspec.com

optek-Danulat, Inc.

262-437-3600

www.optek.com

PicoQuant GmbH

+49-(0)30-639 2-6929

www.picoquant.com

Princeton Instruments

www.princetoninstruments.com

tec5usa

516-653-2000

www.tec5usa.com

TechnoSpex Pte Ltd

+656276 6928

www.technospex.com

Teledyne Princeton Instruments 609-587-9797

princetoninstruments.com

US Fiberoptec Technology Inc.

1 (408) 834-7422

usfiberoptec.com

Ushio America, Inc.

800-838-7446

www.ushio.com

Spectrometers (X-ray)

Amptek Inc.

781-275-2242

www.amptek.com
Fischer Technology, Inc. 860-683-0781

www.fischer-technology.com

IXRF, Inc.

512-386-6100

www.ixrfsystems.com

PNDetector $\mathrm{GmbH}$

+49 89 309087-100

www.pndetector.de

Rigaku Corporation

+1-281-362-2300

www.rigaku.com

Sigray, Inc.

925-446-4183

http://www.sigray.com

\section{Spinning Disk Confocal \\ Microscopy \\ Andor Technology +442890237126 \\ www.andor.oxinst.com \\ CrestOptics \\ www.crestopt.com \\ FEI Munich $\mathrm{GmbH}$ \\ +49898956620 \\ www.fei.com \\ Photometrics \\ 520-889-9933 \\ www.photometrics.com \\ Teledyne Photometrics \\ 520-889-9933 \\ photometrics.com}

\section{SPM Signal Analyzer/Controller SPECS}

$+49-30-4678240$

www.specs.com

\section{Stains}

Agar Scientific Ltd +44 (0)1279 215506

www.agarscientific.com

Nanoprobes, Inc.

1-877-447-6266, (631) 205-9490

www.nanoprobes.com

\section{Stage Automation}

Ealing Catalog

800-295-3220

www.ealingcatalog.com

Haydon Kerk Motion Solutions, Inc. 203-756-7441

www.haydonkerkpittman.com

Hirox-USA, Inc.

201-342-2600

www.hirox-usa.com

Mad City Labs, Inc.

608-298-0855

www.madcitylabs.com

Motion X Corporation

805-968-2001

www.motionxcorp.com

Optimal Engineering Systems, Inc. 888-777-1826

www.oesincorp.com
Prior Scientific, Inc.

781-878-8442

www.prior.com

ResAlta Research Technologies Corp. 303-748-4346

www.resaltatech.com

Velmex, Inc.

585-657-6151

www.velmex.com

Voxa

415-858-0393

http://projectvoxa.com

\section{Stage Micrometers}

APPLIED IMAGE Inc.

585-482-0300

www.appliedimage.com

Ealing Catalog

800-295-3220

www.ealingcatalog.com

Graticules Optics Ltd

+441732360460

www.graticulesoptics.com

HTA Photomask

408 452-5500

www.htaphotomask.com

Ted Pella, Inc.

800-237-3526

www.tedpella.com

\section{Standards for Microanalysis}

APPLIED IMAGE Inc.

585-482-0300

www.appliedimage.com

Avian Rochester, LLC

+1585 259-5956

avianrochester.com

Geller MicroAnalytical Laboratory, Inc. 978-887-7000

www.gellermicro.com

Ted Pella, Inc.

800-237-3526

www.tedpella.com

\section{Stepper Motor Based}

\section{Linear Actuators}

Adsys Controls, Inc.

949-682-5430

www.AdsysControls.com

Haydon Kerk Motion Solutions, Inc. 203-756-7441

www.haydonkerkpittman.com

Optimal Engineering Systems, Inc. 888-777-1826

www.oesincorp.com

\section{Stereo 3D Imaging and}

\section{Measurement}

Digital Surf

0033381504800

www.digitalsurf.com

Edge-3d

808.344.5954

www.edge-3d.com 
Nili Walp

860-355-3776

www.visioneng.us

ResAlta Research Technologies Corp. 303-748-4346 www.resaltatech.com

Vision Engineering, Inc. 860-355-3776 www.visioneng.us

\section{Stereo \& Zoom Microscopes}

Absolute Clarity \& Calibration, LLC 860-583-0502 www.absoluteclarity.com

Capovani Brothers Inc. 518-346-8347 www.capovani.com

Excel Technologies 860-741-3435 www.exceltechnologies.com

Leica Microsystems 800-248-0123 www.leica-microsystems.com

LW Scientific 800-726-7345 www.Iwscientific.com

Martin Microscope Company 864-242-3424 www.MartinMicroscope.com

McCrone Microscopes \& Accessories 630-887-7100 www.mccrone.com

Meiji Techno America www.meijitechno.com

MICROS Produktions-und HandelsgmbH www.micros.at

Mitutoyo America Corp. www.mitutoyo.com

Motic Instruments, Inc. 604-303-9033 https://moticmicroscopes.com/

SCHOTT North America, Inc. Lighting and Imaging

+1-508-765-9744

www.us.schott.com/lightingimaging

Ted Pella, Inc. 800-237-3526 www.tedpella.com

Vee Gee Scientific 800-423-8842 www.veegee.com

Vision Engineering, Inc. 860-355-3776 www.visioneng.us

\section{Stereoscopic Viewing Systems}

TRU-Vu Monitors, Inc. 847-259-2344 www.TRU-VuMonitors.com

Visage Imaging 858-356-5517 www.visageimaging.com

Vision Engineering, Inc. 860-355-3776 www.visioneng.us
Storage Supplies

Bel-Art Products

www.belart.com

General Data Healthcare

844-643-1129

www.general-data.com

Globe Scientific Inc. www.globescientific.com

Ted Pella, Inc. 800-237-3526

www.tedpella.com

\section{Student Microscopes}

Adsys Controls, Inc. 949-682-5430 www.AdsysControls.com

Martin Microscope Company 864-242-3424

www.MartinMicroscope.com

\section{Super Resolution Microscopy}

Abberior Instruments America LLC +15619729300

www.abberior-instruments-america. com

Andor Technology

+442890237126

www.andor.oxinst.com

Axiom Optics

617-221-6636

www.axiomoptics.com

Bruker Fluorescence Microscopy

608-662-0022

http://www.bruker.com

Bruker Nano Surfaces and Metrology

520-741-1044 ext. 1075

www.bruker.com/nano

Forth Dimension Displays Ltd.

+44 0 1383-827-950

www.forthdd.com

Holo/Or Ltd.

+972-8-9409687

www.holoor.co.il/

Mad City Labs, Inc.

608-298-0855

www.madcitylabs.com

NTT Advanced Technology Corporation www.ntt-at.com

Olympus Life Science

781) $419-3687$

www.olympus-lifescience.com

PIEZOCONCEPT

$+33(0) 478742456$

www.piezoconcept.com

Phaseview

+33954030543

www.phaseview.com

PI (Physik Instrumente), LP

508-832-3456

www.pi-usa.us/microscopy

Scientific Volume Imaging +31356421626

ZEISS

1-800-233-2343

www.zeiss.com/microscopy
Supplies

ASI/Applied Scientific Instrumentation 541-461-8181

www.asiimaging.com

Bel-Art Products

www.belart.com

BrandTech Scientific, Inc. 888-522-2726

www.brandtech.com

Duniway Stockroom Corp. 650-969-8811

www.duniway.com

M.E. Taylor Engineering, Inc.

301-975-9798

www.semicro.org

Ted Pella, Inc.

800-237-3526

www.tedpella.com

TGS Technologies, LLC

724-453-3865

www.tgstechnologies.net

\section{Surface Analysis}

Advanced MicroBeam, Inc. 330-394-1255

www.advancedmicrobeam.com

Anderson Materials Evaluation, Inc. 410-740-8562

www.andersonmaterials.com

Applied Spectra, Inc.

510-657-7679

www.appliedspectra.com

Anasys Instruments

805-730-3310

www.anasysinstruments.com

CAMCOR

541-346-6447

www.camcor.uoregon.edu

Digital Surf

0033381504800

www.digitalsurf.com

Eurofins EAG Laboratories 800-366-3867

www.eag.com

Filmetrics, Inc.

858-573-9300

www.filmetrics.com

Geller MicroAnalytical Laboratory, Inc. 978-887-7000

www.gellermicro.com

Hiden Analytical

+44 (0) 1925445225

www. HidenAnalytical.com

Hirox-USA, Inc.

201-342-2600

www.hirox-usa.com

Hysitron, a Bruker Company

952-835-6366

www.hysitron.com

ION-TOF USA, Inc.

845-352-8082

www.iontofusa.com

Kratos Analytical Inc., a Shimadzu Group

Company.

845-426-6700

www.KRATOS.com 
LeRoy Eyring Center for Solid State Science

480-727-8578

https://le-csss.asu.edu

McCrone Associates 630-887-7100

www.mccrone.com

neaspec $\mathrm{GmbH}$ 49-176-2320-3996

www.neaspec.com

Object Research Systems 514-843-3861

www.theobjects.com

Photon etc.

514-385-9555

www.photonetc.com

Physical Electronics

952-828-6100

www.phi.com

Polylnsight LLC

www.polyinsight.com

Renishaw plc.

847-286-9953

www.renishaw.com/raman

SAES Getters USA, Inc.

719-576-3200

www.saes-group.com

SPECS

+49-30-4678240

www.specs.com

TREK, an Advanced Energy Company 1-800-FOR-TREK

www.trekinc.com

WITec GmbH $+49(0) 731140700$

www.witec.de

\section{Surface Profiling}

Applied Electro-Optics, Inc. 512-686-0752

www.aeousa.com

Forth Dimension Displays Ltd. +44 0 1383-827-950 www.forthdd.com

Filmetrics, A KLA Company 858-573-9300

www.filmetrics.com

KEYENCE Corporation of America 888-539-3623

www.keyence.com

LeRoy Eyring Center for Solid State Science

480-727-8578

https://le-csss.asu.edu

Micro Star Technologies

800-533-2509

www.microstartech.com

Object Research Systems

514-843-3861

www.theobjects.com

Zygo Corporation

8603478506

www.zygo.com

\section{Surface Roughness}

Olympus

8322437900

https://www.olympus-ims. com/en/microscope/?utm source=microscopy-today\&utm _ medium=marketing_network\&utm campaign=Dec_2020_IE_All\&utm_ content=listing

\section{Tabletop SEM}

Angstrom Scientific, Inc.

201-962-7222

www.angstrom.us

\section{TEM Accessories}

Circuit Insights LLC

626-201-0488

www.LoopSlooth.com

DigiSENS

www.digisens3d.com

Direct Electron, LP

+1 858-384-0291

www.directelectron.com

E. A. Fischione Instruments, Inc. 724-325-5444

www.fischione.com

Euclid Technologies, LLC

(301) 637-0669

www.euclidtechlabs.com

FEI

503-726-7500

www.fei.com

Gatan

925-463-0200

www.gatan.com

Hummingbird Scientific

360-252-2737

www.hummingbirdscientific.com

Hysitron, a Bruker Company

952-835-6366

www.hysitron.com

ibss Group, Inc.

650-513-1488

www.ibssgroup.com

Integrated Dynamics Engineering IDE 781-326-5700

www.ideworld.com

MiTeGen

1-607-266-8877

www.mitegen.com

NanoMEGAS

480-389-6816

www.nanomegasusa.com

Scientific Instruments \& Applications, Inc.

770-232-7785

www.sia-cam.com

Spicer Consulting Limited

$+44(0) 1234765773$

www.spicerconsulting.com

Ted Pella, Inc.

800-237-3526

www.tedpella.com
TEM Grids

Agar Scientific Ltd +44 (0)1279215 506 www.agarscientific.com

Dune Sciences, Inc. 541-359-1959 www.dunesciences.com

Graticules Optics Ltd

+441732360460

www.graticulesoptics.com

Ladd Research

802-658-4961

www.laddresearch.com

L.A. Giannuzzi \& Associates LLC

321-663-3806

www.LAGiannuzzi.com

NORCADA INC.

780-431-9637

www.norcada.com

SPI Supplies

610-436-5400

www.2spi.com

Ted Pella, Inc.

800-237-3526

www.tedpella.com

YW MEMS

+86-512-6726-6051

http://www.cleansin.com/

\section{TEM Imaging Software}

EMSIS GmbH

+492512979620

www.emsis.eu

Gatan

925-463-0200

www.gatan.com

Image Metrology A/S

+4546923400

www.imagemet.com

Voxa

415-858-0393

http://projectvoxa.com

TEM Imaging Systems

Advanced Microscopy Techniques 978-774-5550

www.amtimaging.com

\section{TEM Sample Handling}

Gatan

925-463-0200

www.gatan.com

Voxa

415-858-0393

http://projectvoxa.com

\section{TEM and SEM Specimen}

\section{Preparation}

3D-Micromac AG

+49371 40043-222

www.3d-micromac.com

CryoSol-World BV

$+31(0) 433022472$

www.cryosol-world.com 


\section{A Guide to Microscopy Products}

Dune Sciences, Inc. 541-359-1959

www.dunesciences.com

EXpressLO LLC

321-663-3806

www.EXpressLO.com

Gatan

925-463-0200

www.gatan.com

L.A. Giannuzzi \& Associates LLC

321-663-3806

www.LAGiannuzzi.com

Leica Microsystems, Inc.

800-248-0123

www.leica-microsystems.com

Microscopy Innovations

715-384-3292

www.microscopyinnovations.com

RMC Boeckeler

1-520-745-0001

www.rmcboeckeler.com

SPI Supplies

610-436-5400

www.2spi.com

\section{Testing Equipment}

Associated Environmental Systems 978.772.0022

www.AssociatedEnvironmentalSystems. com.

ATAGO U.S.A., Inc.

425-637-2107

http://www.atago-usa.com

Berkeley Nucleonics Corp

1-415-453-9955

www.berkeleynucleonics.com

EAG Laboratories

877-709-9526

www.eag.com

Fischer Technology, Inc.

860-683-0781

www.fischer-technology.com

Hysitron, a Bruker Company

952-835-6366

www.hysitron.com

Kammrath \& Weiss

(516) 313-9742

https://www.kammrath-weiss.com/en/

LECO Corporation

269-985-5496

www.leco.com

Mager Scientific, Inc.

734-426-3885

www.magersci.com

Minus K Technology, Inc.

310-348-9656

www.minusk.com

Nanomechanics, Inc., a KLA Tencor

Company

865-978-6490

www.nanomechanicsinc.com

Nextteq LLC

877-312-2333

www.nextteq.com
Pfeiffer Vacuum

603-578-6500

www.pfeiffer-vacuum.com

Psylotech Inc.

+1-847-328-7100

www.psylotech.com

Quantum Composers Inc

406-582-0227

Quantumcomposers.com

SCHOTT North America, Inc. Lighting and Imaging

+1-508-765-9744

www.us.schott.com/lightingimaging

Spectronics Corporation

516-333-4840

www.spectroline.com

Used-Line

845-287-0066

Used-Line.com

Zygo Corporation

8603478506

www.zygo.com

\section{Thin-Film/Flex Circuit}

METRIGRAPHICS, LLC

800-261-2557

http://www.metrigraphicsllc.com/

\section{Thin Film Thickness}

\section{Measurement}

Covalent Metrology

408-498-4611

www.covalentmetrology.com

CRAIC Technologies, Inc.

310-573-8180

www.microspectra.com

Filmetrics, A KLA Company

858-573-9300

www.filmetrics.com

Fischer Technology, Inc.

860-683-0781

www.fischer-technology.com

Kurt J. Lesker Company

412-387-9200

www.lesker.com

LeRoy Eyring Center for Solid State

Science

480-727-8578

https://le-csss.asu.edu

Phillip Technologies

864-277-1560

www.PhillipTech.com

PIKE Technologies, Inc.

608-271-2721

www.piketech.com

Renishaw plc.

847-286-9953

www.renishaw.com/

raman TCG, Inc.

925-240-5210

www.tcg-rep.com

Thin-walled Capillary Tubes

Charles Supper Company

508-655-4610

www.charles-supper.com
Tip Calibrators

Ted Pella, Inc.

800-237-3526

www.tedpella.com

Tips (SPM)

Carbon Design Innovations

650-697-7070

www.carbondesigninnovations.com

Ted Pella, Inc.

800-237-3526

www.tedpella.com

Tissue Processors

Microscopy Innovations 715-384-3292

www.microscopyinnovations.com

PRO Scientific www.proscientific.com

Sonics \& Materials, Inc. 203-270-4600

www.sonics.com

Ted Pella, Inc. 5303395049

www.tedpella.com

Tools

Minitool, Inc.

408-395-1585

www.minitoolinc.com

Ted Pella, Inc.

800-237-3526

www.tedpella.com

Total Internal Reflection

Microscopy (TIFR)

Alluxa, Inc.

855-4Alluxa

www.alluxa.com

FEI Munich $\mathrm{GmbH}$

+49898956620

www.fei.com

LASERGLOW Technologies

+1416-729-7976

www.laserglow.com

Mad City Labs, Inc.

608-298-0855

www.madcitylabs.com

VEROPTICS

1-800-939-6627

www.veroptics.com

Trace Element Analysis Services CAMCOR

541-346-6447

www.camcor.uoregon.edu

Eurofins EAG Laboratories

800-366-3867

www.eag.com

Transmission Electron

Microscopes (TEMs)

Delong America

1-866-335-6648

www.lv-em.com 
EAG Laboratories

877-709-9526

www.eag.com

Electron Optica, Inc.

415-706-1557

www.electronoptica.com

FEl

503-726-7500

www.fei.com

Hitachi High Technologies America, Inc. 800-253-3053

www.hitachi-hightech.com/us

JEOL USA

978-535-5900

www.jeolusa.com

Nion Co.

425-822-1521

www.nion.com

TSS Microscopy

$+15036164710$

http://www.tssmicroscopy.com/

\section{Tweezers}

Bruker Nano Surfaces and Metrology 520-741-1044 ext. 1075

www.bruker.com/nano

Holo/Or Ltd.

+972-8-9409687

www.holoor.co.il/

Ted Pella, Inc.

800-237-3526

www.tedpella.com

Transmission Electron

Microscopy (TEM) Courses

Lehigh Microscopy School

610-758-1112

www.lehigh.edu/microscopy

\section{Ultramicrotomes (See Microtomes \& Ultramicrotomes)}

\section{Ultrasonic Cleaners}

Ted Pella, Inc.

800-237-3526

www.tedpella.com

\section{Used Equipment}

Absolute Clarity \& Calibration, LLC 860-583-0502

www.absoluteclarity.com

Advanced Surface Microscopy, Inc. 317-895-5630 www.asmicro.com

ATAGO U.S.A., Inc. 425-637-2107 http://www.atago-usa.com

Capovani Brothers Inc. 518-346-8347 www.capovani.com

Duniway Stockroom Corp. 650-969-8811 www.duniway.com
Microtome Service Company 315-451-1404

www.microtomeserviceco.com

Scientific Instruments \& Applications, Inc. 770-232-7785

www.sia-cam.com

TCG, Inc.

925-240-5210

www.tcg-rep.com

TSS Microscopy

+15036164710

http://www.tssmicroscopy.com/

\section{Vacuum Equipment}

Advent Research Materials Ltd +44 (0) 1865884440 www.advent-rm.com

BrandTech Scientific, Inc. 888-522-2726 www.brandtech.com

Denton Vacuum 856-439-9111 www.dentonvacuum.com

Duniway Stockroom Corp. 650-969-8811 www.duniway.com

Ellcie Industries $\mathrm{GmbH}$ +493881755400 www.ellcie.com

Gamma Vacuum 952-445-4841 www.gammavacuum.com

HEIDENHAIN Corporation 847-490-1191 www.heidenhain.us

Kimball Physics Inc. 603-878-1616 www.kimballphysics.com

Kurt J. Lesker Company 412-387-9200

www.lesker.com

McPherson Instruments 978-256-4512 www.McPhersonInc.com

MKS Instruments, Inc. 978-645-5500 www.mksinst.com

Nor-Cal Products by Pfeiffer Vacuum 800-824-4166 www.n-c.com

Pfeiffer Vacuum 603-578-6500 www.pfeiffer-vacuum.com

Quorum Technologies, Ltd. $+44(0) 1323810981$ www.quorumtech.com

SAES Getters USA, Inc. 719-527-4119 www.saesgetters.com

Ted Pella, Inc. 800-237-3526 www.tedpella.com

ULVAC Technologies, Inc. 978-686-7550 www.ulvac.com
Vacuum Evaporators

Ladd Research 802-658-4961 www.laddresearch.com

Phillip Technologies 864-277-1560 www.PhillipTech.com

Quorum Technologies, Ltd. $+44(0) 1323810981$ www.quorumtech.com

Ted Pella, Inc. 800-237-3526 www.tedpella.com

Used-Line 845-287-0066

Used-Line.com

\section{Vacuum Oils}

BrandTech Scientific, Inc. 888-522-2726 www.brandtech.com

Duniway Stockroom Corp. 650-969-8811 www.duniway.com

Kurt J. Lesker Company 412-387-9200 www.lesker.com

Ted Pella, Inc. 800-237-3526 www.tedpella.com

\section{Vacuum Tweezers}

Ted Pella, Inc. 800-237-3526 www.tedpella.com

\section{Vibrating Sample}

Magnetometer (VSM)

NanoMagnetics Instruments Ltd. +441865 522989 www.nanomagnetics-inst.com

\section{Vibration Isolation Systems}

AutoMate Scientific

510-845-6283

www.autom8.com

Capovani Brothers Inc.

518-346-8347

www.capovani.com

Herzan, LLC

949-363-2905

www.herzan.com

Integrated Dynamics Engineering IDE 781-326-5700 www.ideworld.com

Janis Research Company, LLC 781-491-0888 www.janis.com

Lake Shore Cryotronics 781-491-0888 www.lakeshore.com/janis

Minus K Technology, Inc. 310-348-9656 www.minusk.com 
TMC

800-542-9725

www.techmfg.com

Vibration Engineering Consultants +1 831-465-9189

https://vibeng.com/

\section{Vibratory Slicers}

Ted Pella, Inc. 800-237-3526

www.tedpella.com

\section{Video Microscopes}

Adsys Controls, Inc. 949-682-5430 www.AdsysControls.com

Alrad Imaging +44 (0)1635 30345 www.alrad.com

BOCK OPTRONICS INC. 416-674-2804 www.bockoptronics.ca

B\&W Tek, Inc. 302-368-7824 www.bwtek.com

Dunwell Tech 888-668-2442 http://dinolite.us/

Hirox-USA, Inc. 201-342-2600 www.hirox-usa.com

ioLight Magnificent Mobile Microscopes +44 (0)333202 7101 ioLight.co.uk

LW Scientific 800-726-7345 www.Iwscientific.com

Mitutoyo America Corp. www.mitutoyo.com

Navitar 585-359-4000 www.navitar.com

TRU-Vu Monitors, Inc. 847-259-2344 www.TRU-VuMonitors.com

Zarbeco, LLC 973-933-2043 www.zarbeco.com

\section{Water Chillers}

Applied Thermal Control Ltd. +441530389998 www.app-therm.com

\footnotetext{
Wavelength Dispersion X-ray Spectrometry (WDS)

Advanced MicroBeam, Inc. 330-394-1255 www.advancedmicrobeam.com

Bruker Corporation 608-276-3000 www.bruker.com

Bruker Nano Analytics 908-256-2627 www.bruker.com/nano-analysis
}

EDAX, Inc. 201-529-4880 www.edax.com

Geller MicroAnalytical Laboratory, Inc. 978-887-7000 www.gellermicro.com

McPherson Instruments 978-256-4512 www.McPhersonInc.com

McCrone Associates 630-887-7100 www.mccrone.com

Thermo Fisher Scientific (508) 479-6623 thermofisher.com $/ \mathrm{msr}$

\section{WDS Detectors \& Systems}

EDAX, Inc.

201-529-4880

www.edax.com

Oxford Instruments America, Inc. 800-447-4717

www.oxford-instruments.com

Thermo Scientific

800-532-4752

www.thermoscientific.com/microscopy

\section{Wide Field Microscopes}

BioTek Instruments, Inc.

888-451-5171

www.biotek.coms

Hirox-USA, Inc.

201-342-2600

www.hirox-usa.com

Mitutoyo America Corp. www.mitutoyo.com

TechnoSpex Pte Ltd +65 62766928 www.technospex.com

\section{Xenon Focused Ion Beam \\ Oregon Physics \\ 503-601-0041 \\ www.oregon-physics.com}

\section{Xenon Lamps}

BLC America

714-889-4116

www.blc-america.com

Bulbworks, Inc.

973-584-7171

www.bulbworks.com

Ushio America, Inc.

800-838-7446

www.ushio.com

UVP LLC

909-946-3197

www.uvp.com

\section{X-ray Analysis Equipment}

AJU Technology 82-53-352-2582

Amptek Inc.

781-275-2242

www.amptek.com
Charles Supper Company 508-655-4610 www.charles-supper.com

DECTRIS Ltd.

+41565002100

www.dectris.com

Fischer Technology, Inc. 860-683-0781

www.fischer-technology.com

Malvern Panalytical

+1 800 279-7297

www.malvernpanalytical.com

MAX Detector Repair Group LLC 608-829-0110 maxdetector.com

Micro Photonics 610-366-7103 www.microphotonics.com

Object Research Systems 514-843-3861 www.theobjects.com

Oxford Instruments America, Inc. 800-447-4717 www.oxford-instruments.com

PNSensor GmbH (49) 8930908713 www.pnsensor.de

Raptor Photonics +442828270141 www.raptorphotonics.com

RaySpec Ltd +44 1628 5330-60 www.rayspec.co.uk

Rigaku Corporation +1-281-362-2300 www.rigaku.com

SAES Getters USA, Inc. 719-576-3200 www.saes-group.com

Sigray, Inc. 925-446-4183 http://www.sigray.com

SII Nanotechnology USA, Inc. www.siintusa.com

Voxa 415-858-0393 http://projectvoxa.com

Xradia, Inc.

925-701-3600

www.xradia.com

\section{X-ray Microscopy}

3D-Micromac AG +49 371 40043-222 www.3d-micromac.com

Deben +44 (0) 1359244 870; 12014105028 www.deben.co.uk

EDAX, Inc. 201-529-4880 www.edax.com

GE Sensing \& Inspection Technologies $\mathrm{GmbH}$

www.ge-mcs.com/en/radiography-xray.html

Micro Photonics 610-366-7103 www.microphotonics.com

Micron Inc. 302-998-1184 https://microanalytical.com 


\section{Products and Services Index}

\section{A Guide to Microscopy Products}

Psylotech Inc. +1-847-328-7100 www.psylotech.com

Rigaku Corporation +1-281-362-2300 www.rigaku.com

Sigray, Inc.

925-446-4183

http://www.sigray.com

Xradia, Inc.

925-701-3600

www.xradia.com

XRE

+32 (0)9 3946794

http://xre.be

ZEISS

1-800-233-2343

www.zeiss.com/microscopy
X-ray Photoelectron

Spectrometers/ Microscopes

Kratos Analytical Inc., a Shimadzu Group

Company.

845-426-6700

www.KRATOS.com

Physical Electronics

952-828-6100

www.phi.com

\section{X-ray Windows}

Abrisa Technologies

+1 (877) 622-7472

www.abrisatechnologies.com

Moxtek, Inc.

801-225-0930

www.moxtek.com
NORCADA INC.

780-431-9637

www.norcada.com

UQG (Lab Glasses)

+ 44 (0) 1223420329

www.uqgoptics.com

YW MEMS

+86-512-6726-6051

http://www.cleansin.com/

\section{Zeta Potential Analyzer}

NanoAndMore USA, Inc. 843-521-1108

www.nanoandmore.com

Malvern Panalytical

+1 800 279-7297

www.malvernpanalytical.com 
